\title{
NRF2 Regulates HER2 and HER3 Signaling Pathway to Modulate Sensitivity to Targeted Immunotherapies
}

\author{
Hilal S. Khalil, ${ }^{1}$ Simon P. Langdon, ${ }^{2}$ Ibrahim H. Kankia, ${ }^{1}$ \\ James Bown, ${ }^{1}$ and Yusuf Y. Deeni ${ }^{1}$ \\ ${ }^{1}$ SIMBIOS, School of Science, Engineering and Technology, Abertay University, Dundee DD1 1HG, UK \\ ${ }^{2}$ Pathology Division, Institute of Genetics and Molecular Medicine, University of Edinburgh, Edinburgh EH4 2XU, UK \\ Correspondence should be addressed to Yusuf Y. Deeni; y.deeni@abertay.ac.uk
}

Received 5 June 2015; Revised 23 August 2015; Accepted 25 August 2015

Academic Editor: Amit Tyagi

Copyright (C) 2016 Hilal S. Khalil et al. This is an open access article distributed under the Creative Commons Attribution License, which permits unrestricted use, distribution, and reproduction in any medium, provided the original work is properly cited.

\begin{abstract}
NF-E2 related factor-2 (NRF2) is an essential transcription factor for multiple genes encoding antioxidants and detoxification enzymes. NRF2 is implicated in promoting cancer therapeutic resistance by its detoxification function and crosstalk with proproliferative pathways. However, the exact mechanism of this intricate connectivity between NRF2 and growth factor induced proliferative pathway remains elusive. Here, we have demonstrated that pharmacological activation of NRF2 by tertbutylhydroquinone (tBHQ) upregulates the HER family receptors, HER2 and HER3 expression, elevates pAKT levels, and enhances the proliferation of ovarian cancer cells. Preactivation of NRF2 also attenuates the combined growth inhibitory effects of HER2 targeting monoclonal antibodies, Pertuzumab and Trastuzumab. Further, tBHQ caused transcriptional induction of HER2 and HER3, while SiRNA-mediated knockdown of NRF2 prevented this and further caused transcriptional repression and enhanced cytotoxicity of the HER2 inhibitors. Hence, NRF2 regulates both HER2 and HER3 receptors to influence cellular responses to HER2 targeting monoclonal antibodies. This deciphered crosstalk mechanism reinforces the role of NRF2 in drug resistance and as a relevant anticancer target.
\end{abstract}

\section{Introduction}

The receptor tyrosine kinases (RTKs) are key drivers of normal cellular proliferation, differentiation, and survival, as well as determinants of cancer initiation, maintenance, and progression [1-4]. Dimerization and stimulation of the intrinsic tyrosine kinase in RTKs lead to the phosphorylation of tyrosine residues in the intracellular domain of the receptors. The phosphotyrosine residues serve as docking sites to recruit a number of signal adapter proteins containing the so-called SH2 and PTB domains, which link RTKs to different cellular signaling pathways such as PI3K/AKT/mTOR, MAPK, and STAT pathways $[5,6]$. Among the RTK superfamily receptors are the type I RTKs that belong to the epidermal growth factor receptor (EGFR or HER) family. The HER receptor network contains four members (HER1, HER2, HER3, and HER4) whose activation kinetics depend significantly on their expression levels which vary across different cells and cancers [7]. Likewise it is these variations combined with receptor interaction that drive and confer complexity in the HER receptor family behaviour.

The two receptors, HER2 and HER3, are nonautonomous and possess certain defining features, in that HER2 has autokinase activity but no known ligands, and HER3 is a pseudokinase receptor that lacks tyrosine kinase activity. These features define the interaction between the HER2 and HER3 receptors and for forming active homodimer and heterodimer complexes. Specifically, mutation or increased gene copy number leads to overexpression of HER2 receptors in cancer cells causing constitutive activation of proliferative pathways in the absence of ligand through homodimerization and RTK autophosphorylation $[1,5,6,10]$. HER2 functions as the shared coreceptor for EGFR, HER3, and HER4 receptors, and these heterodimeric complexes are activated by the partner ligands [11, 12]. Moreover, HER2 is the preferred heterodimerization partner of HER3, whilst HER2 overexpression is believed to enhance the signaling 
from HER3 receptor in response to binding of its specific ligand, neuregulin. As such, HER2-HER3 heterodimers are known to be the strongest elicitors of the PI3K/AKT/mTOR pathway [13-17]. Coexpression of the different receptors, the diversity in their ligand-independent and ligand-dependent activation, variation in their preference towards dimerization partners, and receptor-dependent specificity in cells play a major role in both redundancy in the HER network interaction and effective drug target identification [17-21]. Further complexity in HER2/HER3 activation and signaling arises from the complex transcriptional and posttranslational coregulation of HER2/HER3 receptors and their ligands following HER receptor specific targeted therapies which often lead to inconsistent tumour responses [13, 17, 21].

Nuclear factor- (erythroid-derived 2-) like 2 (NRF2) is a leucine zipper transcription factor and the master regulator of the antioxidant response (AR) pathway. It drives both basal and oxidative stress-induced transcription of a battery of phases I, II, and III detoxification enzymes and cytoprotective genes [22-24] as well as other genes of the metabolic and signal transduction pathways $[14,23,25]$. This is achieved by heterodimerization of NRF2 with small MAF proteins and binding to some genome cis-acting factors called antioxidant response elements (ARE) or electrophile response elements (EpREs) within the promoters of its target genes [26, 27]. Under basal conditions, only a low level of free NRF2 is available in the cytoplasm with some translocating into the nucleus to drive the basal transcription of target genes.

Like the HER receptors [28-31], NRF2 is a recognised agent in cellular proliferation and adaptation to reactive oxygen species (ROS) and in conferring therapeutic resistance to cancers [32-34]. Importantly, NRF2 activation and KEAP1 inactivation mutations leading to permanent constitutive adaptive activation of the NRF2 pathway are frequently observed in cancers [35-37]. Also several therapeutic strategies such as anticancer radio- and chemotherapy greatly depend on ROS manipulation to induce cytotoxicity. Paradoxically, there is a growing body of evidence implicating HER2/HER3, NRF2, and ROS in the promotion of cellular proliferation and therapeutic resistance in cancer cells [38, 39]. Cancer cells have been shown to evolve intricate mechanisms of cellular resistance towards both ROS and other cellular damaging agents as demonstrated by a very robust antioxidant sensing and ROS neutralising mechanisms as well as a highly efficient cytoprotective systems [33, 34, 40-42].

ROS is long not only recognised as the regulator of NRF2 stability and activity but has also been shown to trigger both the AR and the HER family receptor pathways with concomitant transcriptional upregulation of HER2/HER3 complexes and subsequent activation of their functions [30, 31, 43, 44]. Hence, ROS might serve as the point of convergence and as such establish cross relationship between the two pathways. Furthermore, components of the receptor regulated PI3K and MAPK have been shown to regulate NRF2 function [4547], while many aspects of RTK signaling are regulated by ROS whose levels are directly modulated by NRF2 function $[48,49]$.

Since NRF2 is a transcription factor to several hundreds of genes, including proto-oncogenes, it is feasible that
HER2/HER3 receptors are transcriptional targets of NRF2 via direct or indirect means involving ROS. Thus this study aims to investigate this and identify crosstalk between the NRF2 dependent AR pathway and the HER2/HER3 receptors signaling pathway, in order to determine their potential interdependence in eliciting cellular proliferation, cytoprotection, and responses to therapies.

By generating gene transcriptional reporter assays, carrying out pharmacological activation or SiRNA knockdown of NRF2, and performing HER2/HER3 functional inhibition and activation strategies, we have identified a direct node of functional integration of the two pathways in our ovarian cancer cell model which converges at NRF2. We demonstrated that inhibition of NRF2 leads to disruption of the antioxidant pathway and attenuation of HER2/HER3 signaling and that this is as a consequence of transcriptional repression of both HER2 and HER3 genes. Furthermore, we have demonstrated that this functional link could be utilised to either sensitise or reproduce resistant responses in our cell model. Thus, this study reveals a new mechanism of crosstalk between AR and HER2/HER3 pathways and opens up novel avenues of targeting and manipulating the NRF2AR to uncouple and sensitise HER2/HER3 pathways resistant ovarian cancer cells to targeted immunotherapeutics.

\section{Materials and Methods}

2.1. Cell Lines, Culture Conditions, and Treatments. Human ovarian cancer cell lines PEO1 and SKOV3 were maintained in RPMI 1640 media (Gibco Invitrogen) supplemented with $10 \%$ foetal bovine serum (FBS), $2 \mathrm{mM}$ glutamine, $1 \mathrm{mM}$ sodium pyruvate, $100 \mu \mathrm{g} / \mathrm{mL}$ streptomycin, and $100 \mathrm{U} / \mathrm{mL}$ penicillin in an atmosphere of $5 \% \mathrm{CO}_{2}$ and incubated at $37^{\circ} \mathrm{C}$. Before experimental treatments, cells were grown for $24 \mathrm{~h}$ in RPMI 1640 media prepared as above but replacing FBS with $5 \%$ double charcoal stripped FBS (Fisher). Heregulin- $\beta 1$ (HRG, Sigma) was used by preparing $1 \mu \mathrm{mol} / \mathrm{L}$ stock solution made with 5\% trehalose and 10\% FBS in phosphate buffered saline (PBS) and diluted to a final concentration of $1 \mathrm{nmol} / \mathrm{L}$ with media during treatments. Monoclonal antibodies targeting HER2 receptor, Pertuzumab and Trastuzumab (RTKi), were used by directly diluting the drugs in media to a final concentration of $20 \mu \mathrm{g} / \mathrm{mL}$. Tert-butylhydroquinone (tBHQ) stock solution (Sigma) was made with Dimethylsulfoxide (Fisher) and diluted to a final concentration as required with media. For ROS detection, $2^{\prime}, 7^{\prime}$-Dichlorofluorescin diacetate (DCFDA, Sigma) solution was prepared with Dimethylsulfoxide in amber tubes to a concentration of $50 \mathrm{mM}$ and stored at $-20^{\circ} \mathrm{C}$ in the dark until used. For cytotoxicity assay, 3-(4,5-Dimethylthiazol-2-yl)-2,5-Diphenyltetrazolium Bromide (MTT) was needed by making a stock solution of $5 \mathrm{mg} / \mathrm{mL}$ in PBS and filter sterilising it. The solution was stored at $4^{\circ} \mathrm{C}$ in the dark until used.

2.2. Reactive Oxygen Species (ROS) Detection. ROS detection assay was performed by using $2^{\prime}, 7^{\prime}$-Dichlorofluorescin diacetate (DCFDA) staining (Sigma). Briefly, cells were seeded in triplicate at a density of $0.2 \times 10^{5}$ cells/well of opaque 
flat bottom 96-well tissue culture plates in $100 \mu \mathrm{L}$ media without phenol red and allowed to grow for $18 \mathrm{~h}$. Following transfection, cells were washed with PBS and maintained in $100 \mu \mathrm{L}$ of phenol red-free medium and further incubated for $24 \mathrm{~h}$. A $50 \mathrm{mM}$ stock solution of DCFDA was added to each well containing $100 \mu \mathrm{L}$ preexisting media to achieve a final concentration of $25 \mu \mathrm{M}$ and incubated for $45 \mathrm{~min}$ at $37^{\circ} \mathrm{C}$. Fluorescence signal intensities indicating ROS levels were recorded by taking readings using 96-well fluorescent multiplate reader (MODULUS, Promega) using excitation and emission spectra of $485 \mathrm{~nm} / 535 \mathrm{~nm}$. To normalise the fluorescence signal, cells in the same wells were stained with Coomassie brilliant blue stain (Sigma) for $1 \mathrm{~h}$ and washed with distilled water and 10\% sodium dodecyl sulphate (SDS) solution was added to release the absorbed dye for $10 \mathrm{~min}$ while shaking. The absorbance values at $595 \mathrm{~nm}$ were then recorded using a multiplate absorbance reader (MODULUS, Promega) data used after normalising the fluorescence values.

\subsection{Cloning and Expression Vectors Used in the Study.} Closely $1.5 \mathrm{~kb}$ proximal promoter regions of HER2 and HER3 were cloned and used in the current study. The HER2 primer sequences used for each construct were HER2 forward: $5^{\prime}$-GTGCTCGAGGCAAGAAGGGTGCATTTTGAAG-3' and HER2 reverse: $5^{\prime}$-GTCAAGCTTGTCTCTTGGATGGGCCATC- $3^{\prime}$. The HER3 primer sequences used for each construct were HER3 forward: $5^{\prime}$-GTGCTCGAGGCCCTCTAGGTTGCATATCAATAGG-3' and for HER3 reverse: 5'-GTCAAGCTTGAAAAGCAAGCCCAGCAC$3^{\prime}$. For cloning HER2 and HER3 promoters (prHER2 and prHER3, resp.), total genomic DNA was isolated from human cells using DNeasy Blood and Tissue Kit (Qiagen) and quantified using AstraGene microvolume spectrophotometer (AstraNet). $100 \mathrm{ng}$ of the genomic DNA was used to amplify the promoter sequences (MyFi mix, Bioline) using relevant primers that incorporated XhoI and HindIII restriction endonuclease sites $5^{\prime}$ and $3^{\prime}$ ends of the amplified promoters, respectively. PCR conditions for promoter amplification were initial denaturation of $95^{\circ} \mathrm{C}$ for $7 \mathrm{~min}$ followed by 35 cycles of $95^{\circ} \mathrm{C}$ for $30 \mathrm{~s}$ for denaturation, $50^{\circ} \mathrm{C}$ for $30 \mathrm{~s}$ for annealing, and $72^{\circ} \mathrm{C}$ for $90 \mathrm{~s}$ for extension and a final extension for $10 \mathrm{~min}$ at $72^{\circ} \mathrm{C}$. The PCR products were run and extracted from agarose gel (Qiagen), digested using XhoI and HindIII restriction enzymes (Promega), and ligated into PGL3 vector (Promega) to created HER2 and HER2 promoter constructs (prHER2 and prHER3, resp.) driving the expression of luciferase gene for utilisation in dual luciferase reporter assay (Promega). The integrity of cloned sequences was determined by sequencing the plasmids using commercial sequencing service (http://www.dnaseq.co.uk/). All cloned constructs were transfected into relevant cell lines using Lipofectamine 3000 (Life Technologies).

2.4. Protein Extraction and Immunoblotting. For immunoblotting, cells were seeded in $60 \mathrm{~mm}$ tissue culture plates and grown until being $70 \%$ confluent. At the time of protein harvest, cells were trypsinized (Gibco Invitrogen) and washed with PBS. Protein lysates were prepared using radio immune precipitation assay buffer (Pierce Biotech) supplemented with protease and phosphatase inhibitor cocktail (Pierce Biotech) and subjected to sonication of 2 cycles for $10 \mathrm{~s}$ at $50 \%$ pulse. The final mixture was shaken gently on ice for $15 \mathrm{~min}$ and the protein supernatant was obtained following centrifugation of the lysates at $14000 \mathrm{~g}$ for $15 \mathrm{~min}$. Proteins obtained were quantified by Bradford assay (Sigma-Aldrich) using bovine serum albumin as a standard and sample buffer (Nupage LDS, Invitrogen) was added to protein lysates, heated at $70^{\circ} \mathrm{C}$ for $20 \mathrm{~min}$, and stored at $-20^{\circ} \mathrm{C}$ until further use. Once the protein lysates were prepared, they were loaded into wells of 4-12\% gradient SDS-polyacrylamide gels (Nupage Bis-Tris gels, Life Technologies) and subjected to electrophoresis at $200 \mathrm{~V}$ for $1-2 \mathrm{~h}$. Following this, proteins were transferred to polyvinylidene difluoride membranes (PVDF, GE Amersham) using the XCell SureLock Mini-Cell system (Invitrogen) at $50 \mathrm{~V}$ for $90 \mathrm{~min}$ and processed using a commercially available kit (WesternBreeze Chromogenic Immunodetection Kit, Invitrogen). Nonspecific reactivity was blocked by incubation with the blocking reagent supplied in the kit. Membranes were further treated by incubating with primary antibodies (Table 1 ) for $2 \mathrm{~h}$ at room temperature or overnight at $4^{\circ} \mathrm{C}$, followed by incubation for $30 \mathrm{~min}$ at room temperature with appropriate secondary anti-rabbit antibody supplied in the kit. Bands were visualized with the 5Bromo-4-chloro-3-indolyl phosphate/nitroblue tetrazolium chloride based chromogenic substrate. For loading control, immunoblotting of the same lysates was performed using either Beta-actin ( $\beta$-actin) antibody (Abcam Bioscience, UK) or the PVDF membranes with transferred proteins visualised using Ponceau stain (Sigma).

2.5. Luciferase Reporter Assay. For the analysis of promoter activities and transcriptional regulation, the $1.5 \mathrm{~kb}$ promoter regions of HER2 and HER3 genes cloned in pGL3 basic vector (Promega) were transfected into relevant cell lines. Briefly, cells were seeded in triplicate in 24-well plates at a density of $2 \times 10^{5}$ cells per well and allowed to attach for $18 \mathrm{~h}$. Following this, cells were transfected with either $1 \mu \mathrm{g}$ of empty pGL3 basic vector (Promega) or pGL3 basic vector with cloned fragments of HER2 or HER3 promoters driving the expression of luciferase gene, using Lipofectamine 3000 as transfection reagent according to manufacturer's instructions (Life Technologies). Cotransfection was also performed with $0.2 \mu \mathrm{g}$ of pRL-CMV vector (Promega) to provide for an internal control of transfection. Following this, cells were allowed to grow for $24 \mathrm{~h}$, subjected to desired treatments and lysed and protein lysates transferred to opaque white bottom 96-well plates. The dual luciferase activity of fire fly luciferase (from cloned promoters) and Renilla (internal control) in the harvested lysates was measured sequentially by following manufacturer's instructions (Promega) and taking luminescence readings in luminometer (MODULUS, Promega). To determine the transcriptional activity of NRF2 in PEO1 and SKOV3 cell lines, basic pGL3 vector (Promega) containing cloned $8 \mathrm{x}$ cis regulatory ARE promoter elements was transfected into the cell lines grown in 24-well plates and subjected to dual luciferase reporter assay (Promega) as described above. 
TABLE 1: Antibodies used in the study.

\begin{tabular}{llcc}
\hline Antibody & Host & Catalogue number & Company \\
\hline NRF2 & Rabbit & Sc-722 & Santa Cruz \\
Phospho-NRF2 S-15 & Rabbit & ab76026 & Cell Signalling \\
HER2 & Rabbit & 4754 S & Cell Signalling \\
HER3 & Rabbit & $2241 S$ & Cell Signalling \\
Phospho-HER2 T877 & Rabbit & 4060 S & Cell Signalling \\
Phospho-AKT 473 & Rabbit & 2002 & Cell Signalling \\
BID & Rabbit & 4379 & Cell Signalling \\
Phospho-ERK p44/p22 & Rabbit & Sc-10789 & Santa Cruz \\
Heme oxygenase-1 (HO-1) & Rabbit & ab150077 & Abcam \\
Alexa Fluor 488 conjugated secondary antibody & Rabbit & ab175471 & Abcam \\
Alexa Fluor 568 conjugated secondary antibody & Rabbit & &
\end{tabular}

2.6. SiRNA Transfection. Small inhibitory RNA (SiRNA) was used to knockdown NRF2 (Hs_NFE2L2_6, Qiagen). For SiRNA transfection, cells were seeded in triplicate either in 24 -well plates $\left(0.5 \times 10^{5}\right.$ cells $)$, in $60 \mathrm{~mm}$ plates with cells grown on poly-L lysine coated coverslips $\left(0.5 \times 10^{6}\right.$ cells $)$, or in 96-well plates in triplicate $\left(2 \times 10^{4}\right)$ and allowed to grow for $24 \mathrm{~h}$. Following this, cells were cotransfected using either $20 \mathrm{pmol}$ SiRNA and $1 \mu \mathrm{g}$ of different PGL3 promoter constructs (24-well plate) or 75 pmol and 100 pmol SiRNA only ( $60 \mathrm{~mm}$ plates) or $7 \mathrm{pmol}$ of SiRNA (96-well plate) and incubated for a further $24 \mathrm{~h}$. Cells transfected in 24 -well plate were further processed for dual luciferase assay and those in $60 \mathrm{~mm}$ plates were harvested for immunoblotting or used for imaging analysis while those in 96-well plates were processed for cytotoxicity assay. In all cases, scrambled SiRNA was used as a control while transfection was performed using Lipofectamine 3000 (Life Technologies) according to manufacturer's instructions.

2.7. Cytotoxicity Assay. For cytotoxicity (or cell viability) assay, cells were seeded in triplicate at a density of $0.5 \times$ $10^{4}$ cells in 96-well plate and allowed to attach for $18 \mathrm{~h}$. On the day of treatment, old media were removed and $80 \mu \mathrm{L}$ of media containing relevant drugs was added and the plate was incubated for the required period of time. On the day of assay, $20 \mu \mathrm{L}$ of the $5 \mathrm{mg} / \mathrm{mL}$ MTT stock was added to each well and plate was further incubated for $4 \mathrm{~h}$. Following this, the old media with MTT were removed, cells were gently washed with prewarmed PBS, and $100 \mu \mathrm{L}$ of DMSO was added to solubilise the internalised MTT by shaking over an orbital shaker for $15 \mathrm{~min}$. Absorbance of the released dye was measured and recorded using multiplate reader (MODULUS, Promega) at $540 \mathrm{~nm}$.

2.8. Immunocytochemistry/Immunolabelling. For immunocytochemistry, exponentially growing cells were seeded at a density of $5 \times 10^{4}$ cells in complete media onto polyL lysine (Sigma-Aldrich) coated cover slips placed in a 12-well tissue culture plates. Next day, following relevant treatments, cells were washed three times with ice cold PBS and fixed in 3.5\% paraformaldehyde in a standard PBS at room temperature for $30 \mathrm{~min}$. Following this, cells were gently washed twice with $1 \mathrm{~mL}$ of PBS, permeabilized with $0.3 \%$ Triton X-100 for $10 \mathrm{~min}$, and, following three washes with PBS, blocked with a solution containing $1 \%$ goat serum, $1 \%$ bovine serum albumin, and $0.05 \%$ Triton X-100 in PBS for $30 \mathrm{~min}$. Cells were then incubated with relevant primary antibody (Table 1) diluted in blocking solution for $1 \mathrm{~h}$, washed three times with $0.1 \%$ Triton X-100/PBS for $5 \mathrm{~min}$, and then incubated with Alexa Fluor 488 or 568 conjugated goat antirabbit (Table 1) for $30 \mathrm{~min}$. After subsequent three washes with the $0.1 \%$ Triton X-100 in PBS for $5 \mathrm{~min}$, cover slips with cells were mounted on slide using $4^{\prime}, 6$-Diamidino-2Phenylindole, Dihydrochloride (DAPI) containing mounting reagent (Life Technologies) and imaged under relevant filters with a Leica DMiRe2 electronic microscope.

2.9. Statistical Analysis. All statistical analyses were performed using statistical software SPSS (IBM, version 22). Test for normality of data was determined by Shapiro-Wilk and Kolmogorov and Smirnov tests. The significance ( $p$ value) of differences of pooled results was determined by either independent $t$-tests or One WAY ANOVA followed by post hoc Tukey's tests. Significance was defined as $*=p<0.05$, $* *=p<0.01$, and $* * *=p<0.001$.

2.10. Imaging and Analysis. Quantitative analysis of raw immunoblots was performed by capturing the images in high resolution TIFF format files using a charge-coupled-device camera (AxioCam MRc, Carl Zeiss) and subjected to Gelpro analysis software, version 3.1 (Gelpro Media Cybernetics) for integrated optimal densitometry. Fluorescence images of immunocytochemistry were collected under relevant excitation and emission filters depending on the fluorotype under Leica DMiRe2 electronic microscope equipped with iXonEM +897 EMCCD camera (ANDOR Technologies Ltd.). Images were analysed using multidimensional microscopy software Andor Module iQ Core. Colocalization assay was performed and determined with software integral features supplied by Andor iQ Core software. Data were generally expressed as mean \pm S.D. for individual sets of experiments. 


\section{Results}

3.1. Pharmacological Activation of NRF2 Enhances Ovarian Cancer Cell Growth and Protects from Cytotoxicity Caused by HER2-Targeted Immunotherapeutic Agents. Numerous studies have shown that NRF2 promotes resistance to chemotherapeutic agents $[50,51]$ and contributes to general cytoprotection, metabolic reprograming, and cell survival [5255]. On the other hand, targeted immunotherapy involving inhibitory monoclonal antibodies against HER2 receptor has generated interest in recent years as a potential strategy to overcome ovarian cancer cell therapeutic resistance [17, 56]. Using HER 2 overexpressing and low expressing ovarian cancer cell lines SKOV3 and PEO1, respectively [57], we first examined whether preactivation of NRF2 would change the cytotoxic responses of these cells to HER2-targeted immunotherapeutic agents Pertuzumab and Trastuzumab. For this, cells were grown in media containing 5\% charcoal stripped FBS and $1 \mathrm{nmol} / \mathrm{L}$ Heregulin (HRG), a ligand for the HER3 receptor [58] for relevant treatments. Firstly, we found that pharmacological activation of NRF2 by tBHQ alone was sufficient to enhance the proliferation of both cell lines for six days (Figure 1). On the other hand and as expected, exposure of cells to HER2 inhibitors, Pertuzumab and Trastuzumab, inhibited the proliferation of both cell lines for up to 4 days of treatment, while losing its inhibitory effect on day 6. Interestingly, pretreatment of cells with $200 \mu \mathrm{M}$ tBHQ for $5 \mathrm{~h}$ before the introduction of the HER2 inhibitors significantly protected cells from the inhibitory action of the subsequently added HER 2 targeting monoclonal antibodies. This was consistent for both cell lines and for all the treatment days tested (Figure 1). Furthermore, inclusion of tBHQ with the inhibitors not only protected the cells but increased survival even beyond the untreated levels on days 2, 4, and 6 in PEO1 and days 1, 2, and 6 in SKOV3 cell lines (Figure 1). This demonstrated that NRF2 activation is not only implicated in resistance to genotoxic agents as previously demonstrated [55] but can also lead to resistance to immunotherapies involving Pertuzumab and Trastuzumab whose actions otherwise are very specific to HER2 receptors and unrelated to antioxidant pathway.

\subsection{TBHQ Treatment Causes Protein Induction of HER2 and} HER3 and Parallel Increase in Phospho-AKT S473. Previous studies have examined the crosstalk between growth promoting MAPK and PI3K pathways and NRF2 antioxidant pathway in numerous cell systems. However, in the majority of such studies, the focus was regulation of NRF2 by these kinases $[46,47,59,60]$. The observation that preactivation of NRF2 led to resistance against agents of targeted therapy (Figure 1) suggested potential regulation of HER2 dependent growth pathways by NRF2. Hence, we next exposed PEO1 and SKOV3 cell lines to a single concentration of tBHQ for $4 \mathrm{~h}$ and examined the effects of such treatment on the protein levels of HER 2 and HER3 and their downstream substrate pAKT Ser 473. Firstly, higher levels of HER2 receptor were confirmed in SKOV3 cell line that was also accompanied by induced basal pAKT, consistent with previous reports [61]. Secondly, following tBHQ treatment, we saw induction of total HER2 and parallel consequential induction of pATK levels in both cell lines. Further, HER3 was found to decrease in PEO1 while being induced in SKOV3, demonstrating a differential regulation of the receptors in the two cell lines (Figures 2(a) and 2(b)). This is consistent with the increased proliferation seen following tBHQ treatment, as an enhanced cell surface expression of receptors would lead to a greater degree of binding to their HRG ligand and triggers growth promoting signaling.

To explore further HER2 and pAKT induction by tBHQ at a single cell level, we performed subcellular localisation by fluorescent double immunolabelling of these proteins in PEO1 and SKOV3 cell lines following the same treatments (Figure 2(c)). Consistent with Figure 2(a), we saw higher expression of HER2 in SKOV3 as compared to PEO1. For both cell lines, pAKT was found uniformly distributed in the cytoplasm and nucleus. This could be indicating the constitutively active nature of this pathway and could be explained by the presence of HRG in the media. Following tBHQ treatment for $4 \mathrm{~h}$, we saw an increase in HER2 expression and an accompanying increase in pAKT levels as well. Superimposition and colocalisation of the images captured in the red and green fluorescence channels to indicate HER2 and pAKT, respectively, were performed and showed increased localisation of the two proteins, as demonstrated by the appearance of yellow fluorescence following $\mathrm{tBHQ}$ as compared to untreated controls (Figure 2(c)). To confirm and measure the enhanced colocalisation following treatments, we also performed further imaging analysis by generating cytofluorograms and found that Pearson's coefficient of correlation $(r)$ increased in both cell lines following tBHQ exposure (Figure 2(d)). Altogether, these data illustrated effects of tBHQ treatment on RTK mediated growth signaling. This was demonstrated by induction of HER2 and HER3 and activation of AKT following pharmacological activation of NRF2, which supported the enhanced proliferation seen before (Figure 1).

\subsection{Pharmacological Activation of NRF2 Causes Transcrip-} tional Induction of HER2 and HER3 Genes. Previous studies have shown transcriptional perturbation of HER2 and HER3 following different targeted therapy treatments [17]. In some contexts, this was proposed to be used as a biomarker for treatment response [61]. After finding the modulatory effects of tBHQ treatment on protein levels of HER2 and HER3 receptors, we next wanted to identify the mechanism of this upregulation. Specifically, we wanted to examine whether the protein inductions seen in Figure 2 result from transcriptional regulation. To determine this, we generated transcriptional reporter assays for both HER2 and HER3 receptors. This involved developing HER2 and HER3 promoter driven luciferase reporter system (named prHER2 and prHER3, resp.). We transfected these luciferase reporter systems carrying $1.5 \mathrm{~kb}$ of the upstream promoter regions of the two receptors into both PEO1 and SKOV3 cell lines to first determine their basal level of transcription and then studied the effects of tBHQ treatment. Figure 3(a) interestingly revealed that SKOV3 cell line exhibited enhanced basal transcription of both HER 2 and HER3 genes as compared to 

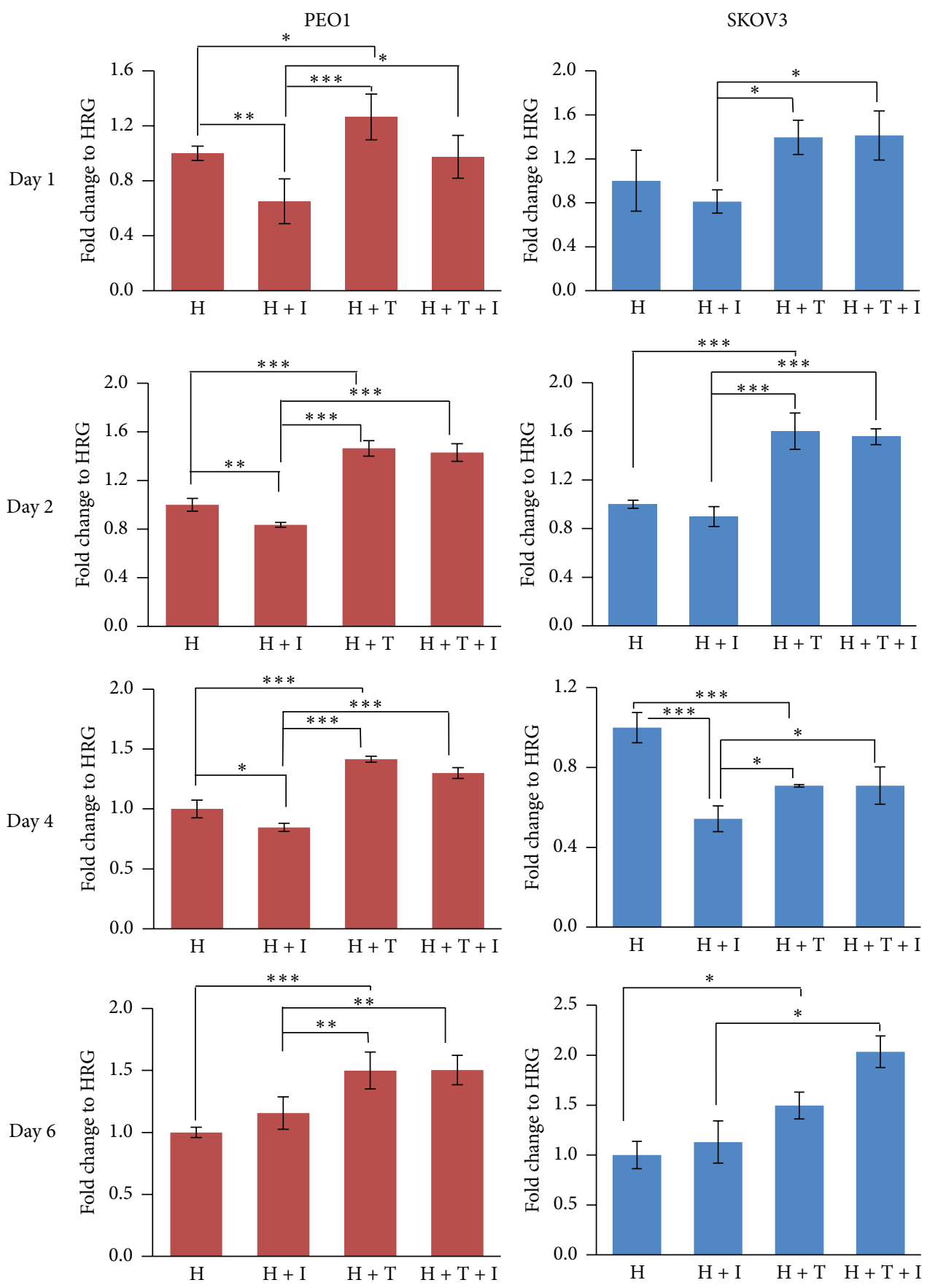

FIgURE 1: NRF2 activation causes cytoprotection from HER2-targeted agents, Pertuzumab and Trastuzumab. PE01 or SKOV3 cells in the presence of $1 \mathrm{nM} H R G$ were either left untreated $(\mathrm{H})$ or treated with $20 \mu \mathrm{g} / \mathrm{mL}$ of HER2 inhibitors Pertuzumab and Trastuzumab $(\mathrm{H}+\mathrm{I})$, $200 \mu \mathrm{M}$ tBHQ $(\mathrm{H}+\mathrm{T})$, or combination of inhibitors and tBHQ $(\mathrm{H}+\mathrm{T}+\mathrm{I})$. TBHQ was added $5 \mathrm{~h}$ in advance. Cell number was assessed indirectly by use of the MTT assay. Values shown are means \pm S.D. of triplicates normalised to untreated controls expressed as 1 . Statistical significance was calculated between $\mathrm{H}+\mathrm{I}, \mathrm{H}+\mathrm{T}$, and $\mathrm{H}+\mathrm{T}+\mathrm{I}$ groups by ONE WAY ANOVA followed by Tukey's post hoc test according to the scale $*: p<0.05, * *: p<0.01$, and $* * *: p<0.001$.

PEO1 cell line (Figure 3(a)). However, the previous western blot analysis in Figure 2 showed higher basal levels of HER3 in PEO1 as compared to SKOV3 whereas HER2 levels were consistently higher in SKOV3. This illustrated that the overexpression of HER2 in SKOV3 cell line could be explained by both gene amplification [62] and higher basal transcription.
We next exposed cells transfected with the prHER2 and prHER3 reporter assays to increasing concentrations of tBHQ to further explore the nature of this transcriptional regulation. Strikingly, both PEO1 and SKOV3 cell lines exhibited significant dose-dependent transcriptional induction of HER2 (Figure 3(b), blue bars). Interestingly, prHER3 


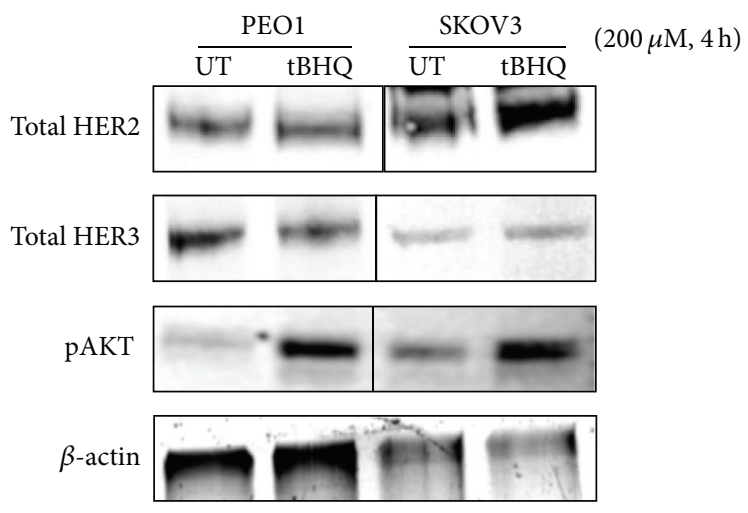

(a)

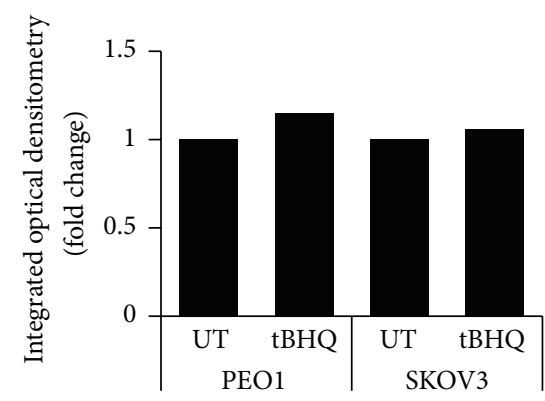

- Total HER2

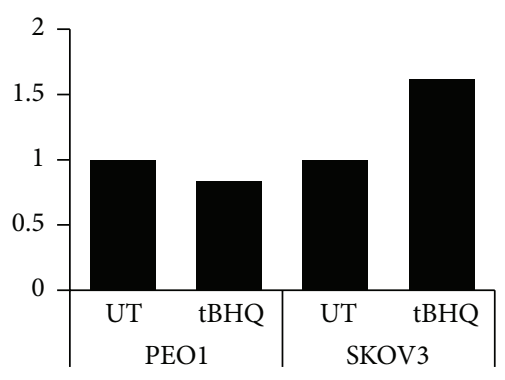

- Total HER3

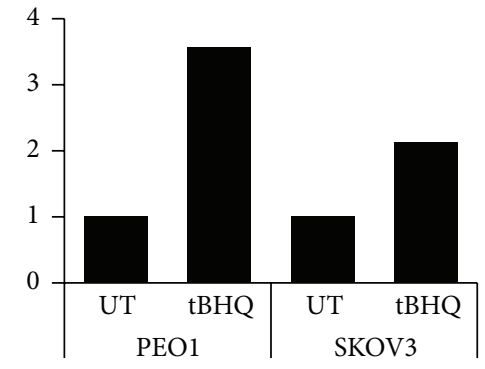

a pAKT

(b)
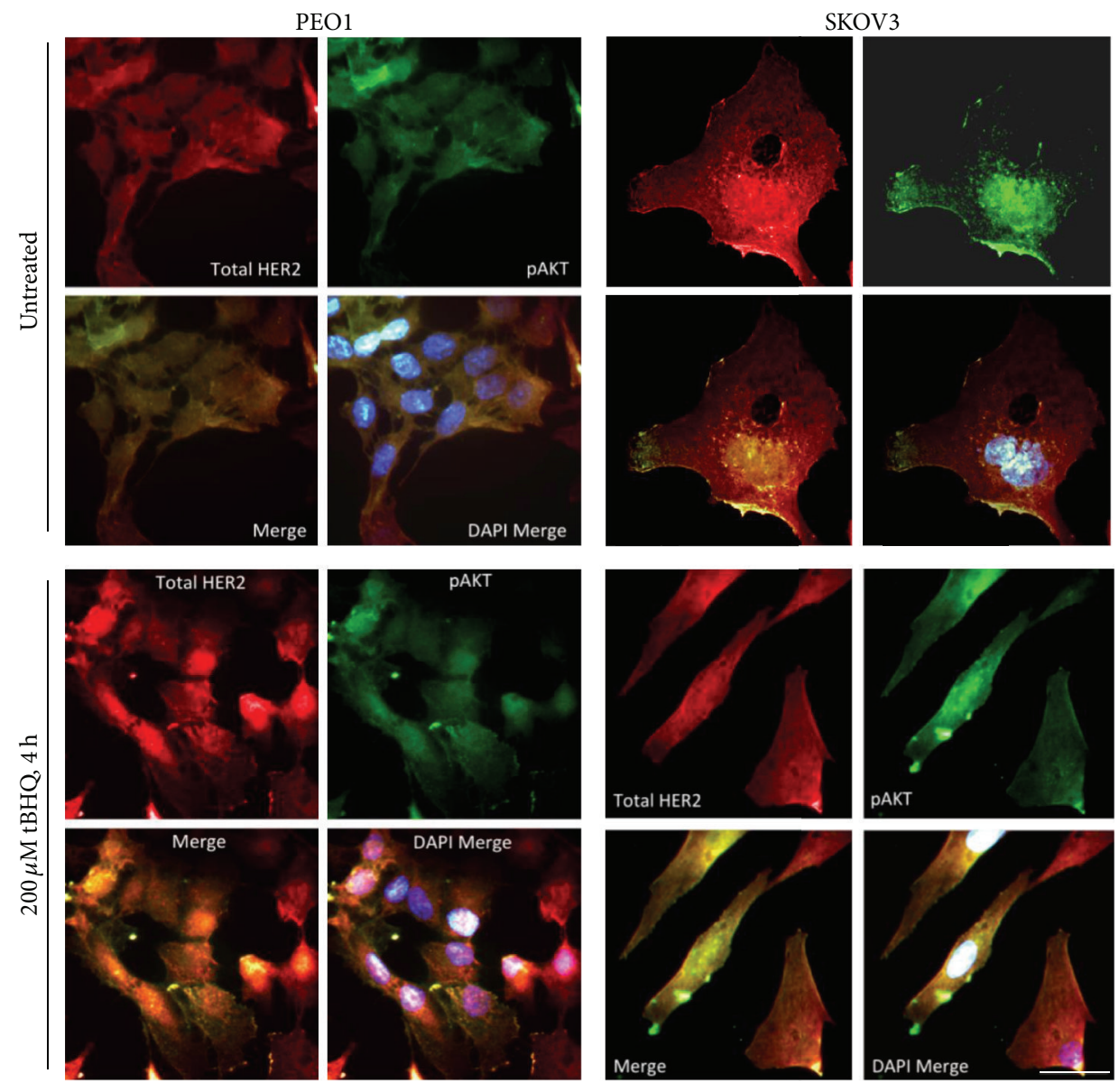

(c)

Figure 2: Continued. 


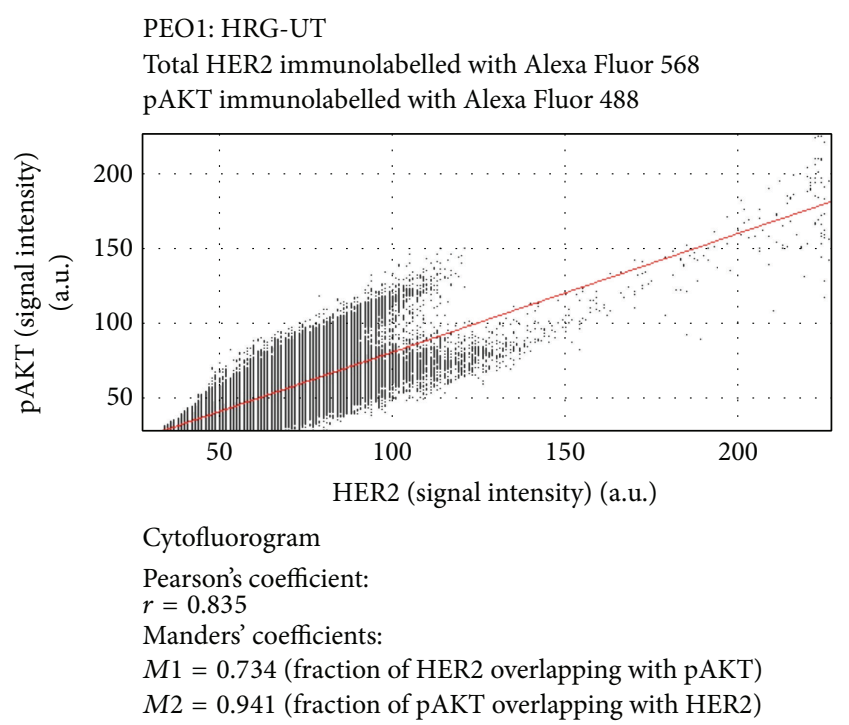

SKOV3: HRG-UT

Total HER2 immunolabelled with Alexa Fluor 568

pAKT immunolabelled with Alexa Fluor 488

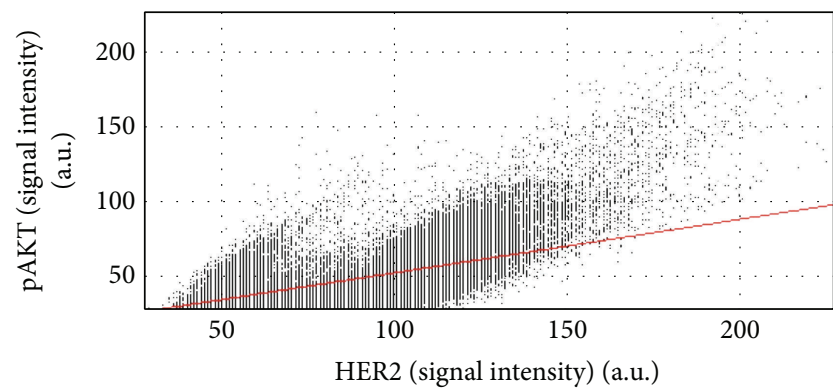

Cytofluorogram

Pearson's coefficient:

$r=0.756$

Manders' coefficients:

$M 1=0.42$ (fraction of HER2 overlapping with pAKT)

$M 2=0.959$ (fraction of pAKT overlapping with HER2)

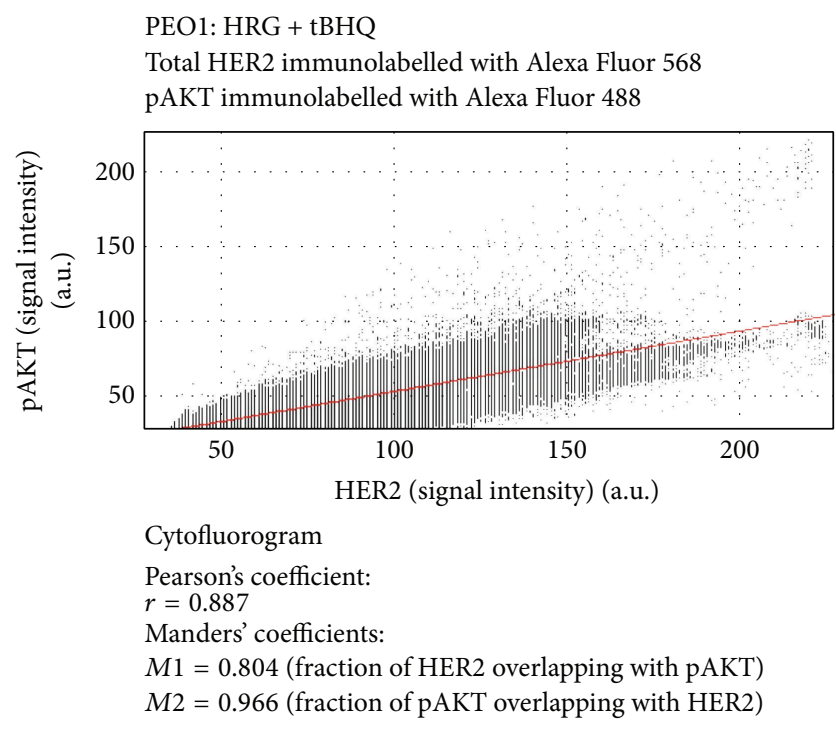

SKOV3: $\mathrm{HRG}+\mathrm{tBHQ}$

Total HER2 immunolabelled with Alexa Fluor 568

pAKT immunolabelled with Alexa Fluor 488

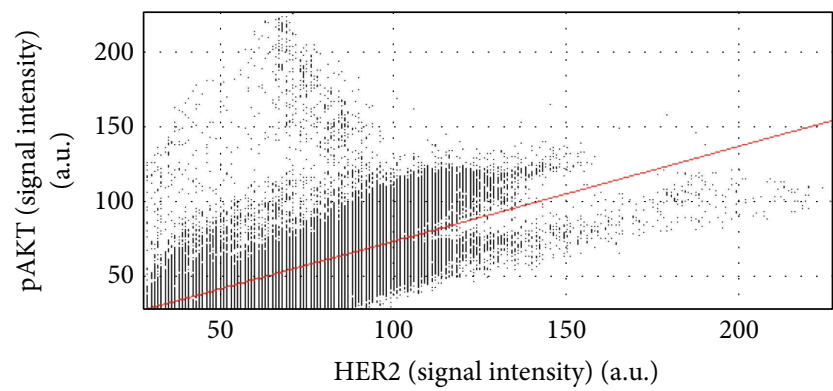

Cytofluorogram

Pearson's coefficient:

$r=0.823$

Manders' coefficients:

$M 1=0.571$ (fraction of HER2 overlapping with pAKT)

$M 2=0.945$ (fraction of pAKT overlapping with HER2)

(d)

FIGURE 2: TBHQ treatment causes protein induction of HER2 and HER3 and upregulation of pAKT levels in PEO1 and SKOV3 cells. (a) Immunoblot analysis following treatment with tBHQ demonstrated protein induction of both HER2 and HER3 receptors and increase of pAKT. Exponentially growing cells were either left untreated (UT) or treated with $200 \mu \mathrm{M}$ tBHQ for $4 \mathrm{~h}$ before being harvested and processed for immunoblotting using relevant antibodies (Table 1). (b) Bar chart showing total HER2, total HER3, and phospho-AKT levels in PEO1 and SKOV3 cell lines by quantifying immunoblot signal intensities obtained in (a) and normalised to the value of UT and expressed as fold change. (c) Immunofluorescent labelling of endogenous HER2 and phospho-AKT reveals protein induction following tBHQ treatment. Cells were processed for immunocytochemistry and immunolabelled using anti HER2 (red fluorescence) or phospho-AKT (green fluorescence) primary antibodies followed by Alexa Fluor conjugated secondary antibodies. Nuclear reference was provided by costaining with $4^{\prime}, 6$-Diamidino-2Phenylindole, Dihydrochloride (DAPI). Scale bar indicates $10 \mu \mathrm{m}$. (d) Analysis of colocalisation between immunostained HER2 and pAKT in the images obtained in (c). Spatial correlation between the two fluorescent signals was obtained by generating cytofluorograms and performing Pearson's correlation analysis.

exhibited a varying response. While prHER3 activity was significantly induced in PEO1 following $50 \mu \mathrm{m}$ tBHQ, increasing dosage beyond $50 \mu \mathrm{M}$ led to its repression. In SKOV3 cells on the contrary, $50 \mu \mathrm{M}$ tBHQ repressed prHER3 activity while increasing dosage of 100 and $200 \mu \mathrm{M}$ led to subsequent induction (Figure 3(b), brown bars). This complex regulation of HER3 is reminiscent of recent reports that revealed that induction of HER2 might repress HER3 expression while its inhibition could lead to transcriptional induction of HER3 $[13,14,63]$. This set of results confirmed the transcriptional basis of induction of HER2 and HER3 protein levels, which concomitantly also led to pAKT induction. 


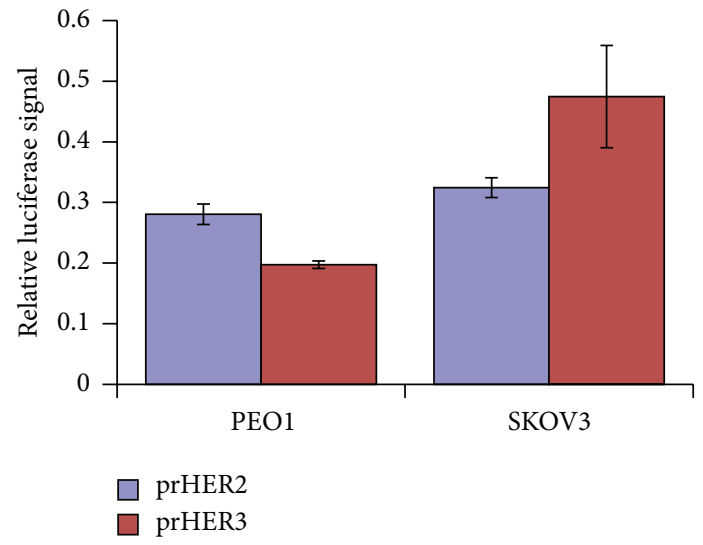

(a)
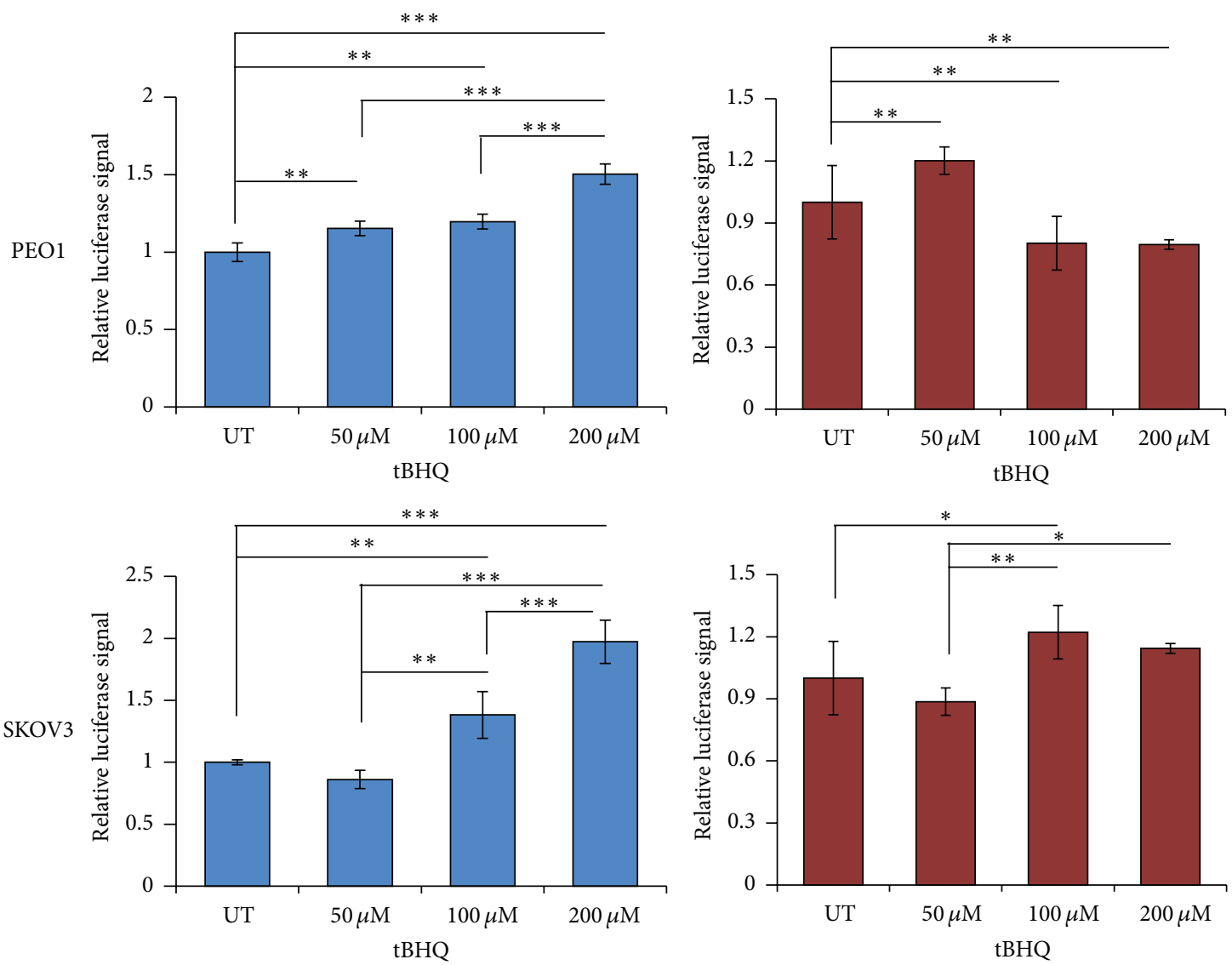

prHER2

prHER3

(b)

FIgURE 3: Nrf2 activation leads to transcriptional induction of HER2 and HER3 in a concentration dependent manner. (a) SKOV3 cells exhibit higher basal transcription of both HER2 and HER3. PEO1 and SKOV3 cells were transfected with either empty PGL3 basic vector or $1 \mu \mathrm{g}$ PGL3 basic vector with cloned $1.5 \mathrm{~kb}$ fragments of either HER2 (prHER2) or HER3 (prHER3) promoter driving the expression of luciferase gene. Cotransfection with $0.2 \mu \mathrm{g}$ pRL-CMV plasmid was performed as an internal transfection control. (b) TBHQ causes transcriptional induction of HER2 and HER3 in a concentration dependent manner. PEO1 and SKOV3 cell lines were transfected in triplicate as in (a) but were treated with different concentrations of $\mathrm{tBHQ}$ as indicated for $4 \mathrm{~h}$. Data shown are the means \pm S.D. of triplicates, normalised to untreated (UT) controls and expressed as fold change with statistical significance determined by ONE WAY ANOVA followed by Tukey's post hoc test $(*: p<0.05, * *: p<0.01$, and $* * *: p<0.001)$. 
3.4. NRF2 Activation Desensitises RTK Signaling Pathway to Combination of HER2 Targeting Monoclonal Antibodies Pertuzumab and Trastuzumab. The observation that tBHQ treatment led to transcriptional induction of HER2 and HER3 suggests that NRF2 may be directly involved in regulating the receptor expression and as such may influence responses to targeted therapies involving HER2 inhibitors. This important question was next investigated by treating PEO1 or SKOV3 cells either with the combination of Pertuzumab and Trastuzumab alone or by cotreatment with $\mathrm{tBHQ}$ to examine the consequences of NRF2 activation on drug responses. Interestingly, some features of the signaling response were similar between these two cell lines while others were more distinct. In the PEO1 cell line, treatment with inhibitors alone induced both HER2 and HER3 levels consistent with the parallel increase in phospho-HER2 T877 (Figure 4) in this cell line. In contrast, for SKOV3, only HER3 expression showed a minor induction while total HER2 levels were reduced explaining the decrease in phospho-HER2 T877 levels as well. In order to better understand the effect of these inhibitors on RTK signaling, we normalised the blot signal of phospho-HER2 in both cell lines to the corresponding values of total HER2 (Figure 4, blue bars). This analysis interestingly revealed that while the inhibitors reduced the ratio of phospho-HER2 to total HER2, cotreatment with tBHQ restored the ratio back to that of untreated controls. This effect was more pronounced in the SKOV3 cell line. Importantly, in terms of pAKT S473, while $4 \mathrm{~h}$ treatment with inhibitors led to minor repression of its levels as revealed by the densitometry analysis, cotreatment with $\mathrm{tBHQ}$ protected this repression and increased pAKT levels beyond that of untreated controls (Figures 4(a) and 4(b)). These results revealed that $\mathrm{tBHQ}$ can protect RTK signaling against the inhibitory action of the drugs. We also included phosphoERK p44/p22 levels in our analysis as ERK was previously shown to be inhibited by drugs targeting HER2 receptor [56]. We saw a very minor repression of phospho-ERK levels only in SKOV3 cells following $4 \mathrm{~h}$ of inhibitor treatment. However, tBHQ dependent induction for ERK was seen in PEO1 cells. Inhibitor treatment did not influence pNRF2 S15 levels in either of the cell lines, but as expected, its levels increased following tBHQ treatment. Finally, we examined intact and cleaved levels of proapoptotic protein BID in order to further support our conclusions drawn from Figure 1. By determining the ratio of cleaved BID over intact, we observed that while treatment with inhibitors induced levels of cleaved BID, tBHQ cotreatment led to a minor repression, further explaining the cytoprotective effect of tBHQ treatment (Figure 4). These results revealed important consequences of tBHQ treatment on targeted therapy using HER2targeted monoclonal antibodies and showed that treatment with NRF2 activator attenuated the inhibitory action of these monoclonal antibodies.

3.5. Knockdown of NRF2 by Small Inhibitory RNA (SiRNA) Elevates ROS, Represses pNRF2 and Heme Oxygenase-1 (HO1) Levels, and Disrupts tBHQ Dependent Induction of ARE. In order to confirm the direct role of NRF2 in $\mathrm{HBHQ}$ dependent induction of HER2 and HER3 receptors, we next knocked down NRF2 using SiRNA. To this end, we first optimized and verified sufficient knockdown of NRF2 using specific SiRNA and then studied the effects of this knockdown on antioxidant pathway. As shown in Figure 5(a), 75 pmol of NRF2 SiRNA produced maximum depletion of NRF2 both following 24 and $48 \mathrm{~h}$ of transfection, while $100 \mathrm{pmol}$ showed lesser depletion (Figure 5(a), black bars indicating band intensities). We next determined whether this depletion is sufficient to cause repression of the antioxidant pathway by examining NRF2 substrates. We found that 75 pmol SiRNA sufficiently downregulated phospho-NRF2 and HO-1 levels as well (Figure 5(b)). Efficiency of internalisation of NRF2 targeting SiRNA in SKOV3 cells using different amounts was confirmed and verified (Figure 5(c)). We next quantified total basal ROS following NRF2 knockdown to determine whether NRF2 depletion caused elevation of ROS. Loading of cells with $2^{\prime}, 7^{\prime}$-Dichlorofluorescin diacetate dye which is a fluorescent marker of intracellular ROS confirmed elevation of ROS resulting from NRF2 knockdown (Figure 5(c)). Finally, we performed immunostaining of endogenous pNRF2 and HO-1 following transfection with either scrambled or NRF2 targeting SiRNA and as consistent with Figure 5(b), we verified repression of pNRF2 and HO-1 levels at single cell level (Figure 5(d)). Having confirmed the effectiveness of our SiRNA-mediated NRF2 knockdown, we next examined whether depletion of NRF2 would also disrupt tBHQ dependent induction of antioxidant pathway in PEO1 and SKOV3 cell lines and thus confirm the direct involvement of NRF2 in this mechanism. To do this, we exposed cells to tBHQ either in the presence of endogenous NRF2 or following its genetic depletion. Figure 6(a) revealed that NRF2 protein induction seen in tBHQ treatment was disrupted following its SiRNA transfection. Next, in order to confirm that NRF2 depletion also caused inhibition of transcriptional antioxidant response program and to further confirm the conclusions drawn from Figure 5(b), we transfected cells with cis-antioxidant response elements (ARE) in luciferase reporter vector driving the expression of luciferase to report transcriptional activity of NRF2. Firstly, we saw repression of ARE signal supporting our conclusions drawn from Figure 5. Secondly, we saw that the $\mathrm{tBHQ}$ treatment regime that had caused induction of NRF2 protein levels (Figure 6(a)), and those of NRF2 substrate HO-1 (Figure 5(b)) and HER2 and HER3 receptor expressions (Figures 2 and 3), also significantly enhanced the activity of the NRF2 dependent antioxidant transcriptional programme in both PEO1 and SKOV3 cell lines (Figure 6(b)). Finally, we saw that such induction was inhibited following knockdown of NRF2 to significant levels as compared to tBHQ treatment alone. Altogether, Figures 5 and 6 provide evidence of knockdown of NRF2, repression of the antioxidant response pathway, and disruption of $\mathrm{tBHQ}$ mediated pathway induction.

3.6. NRF2 Depletion Causes Transcriptional Inhibition of HER2 and HER3 Leading to Repression of HER2, HER3, and pAKT Proteins and Sensitisation to Targeted Immunotherapeutics. As shown in the previous sections, NRF2 activation by $\mathrm{tBHQ}$ not only induced the NRF2 dependent antioxidant response pathway as expected, but surprisingly also induced 


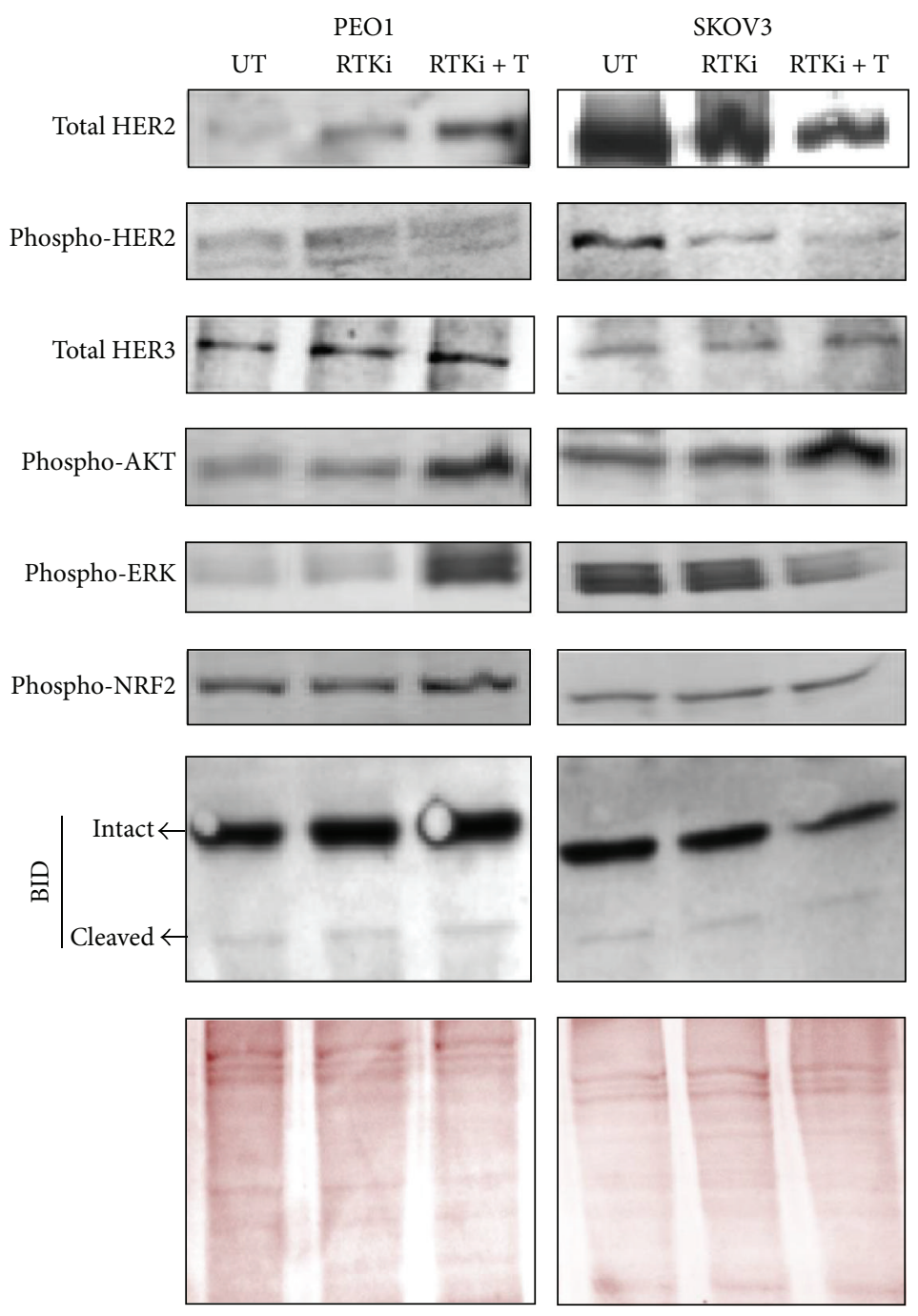

(a)

PEO1

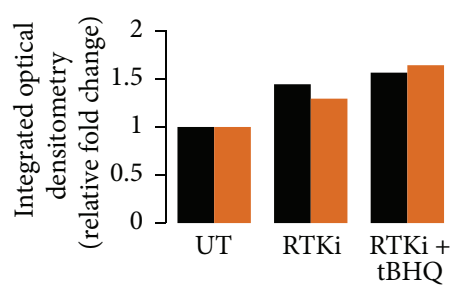

SKOV3

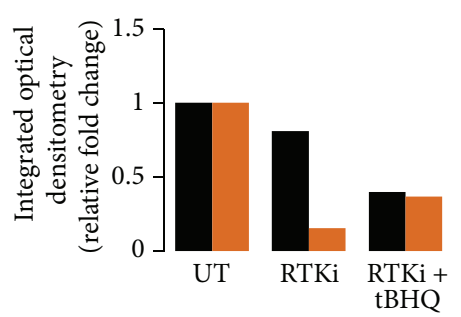

- HER2

pHER2
PEO1

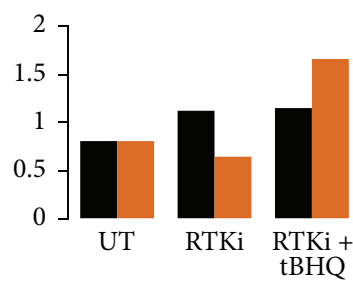

SKOV3

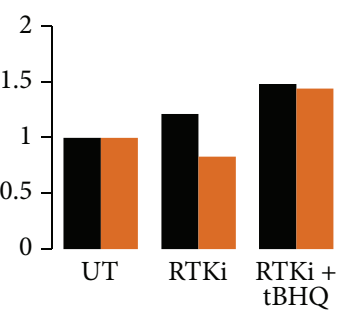

- HER3

pAKT
PEO1

PEO1
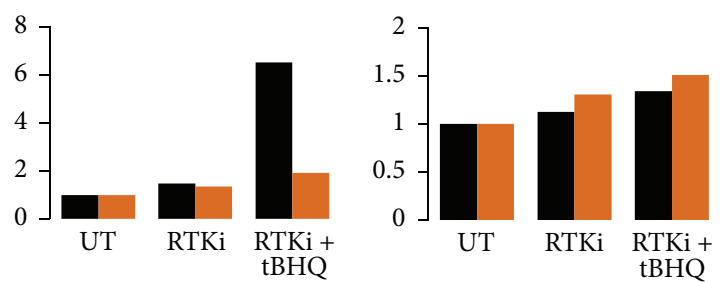

SKOV3

SKOV3
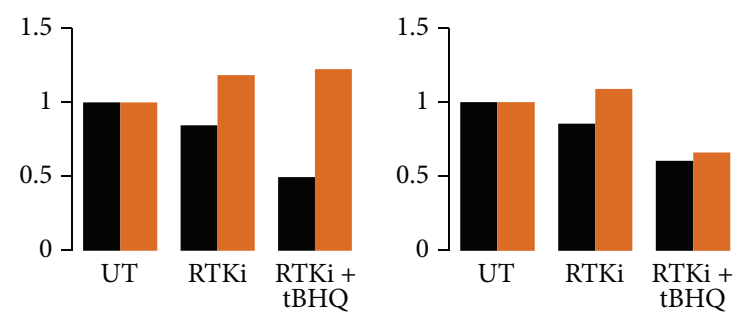

pERK

pNRF2
Intact BID

Cleaved BID

Figure 4: Continued. 

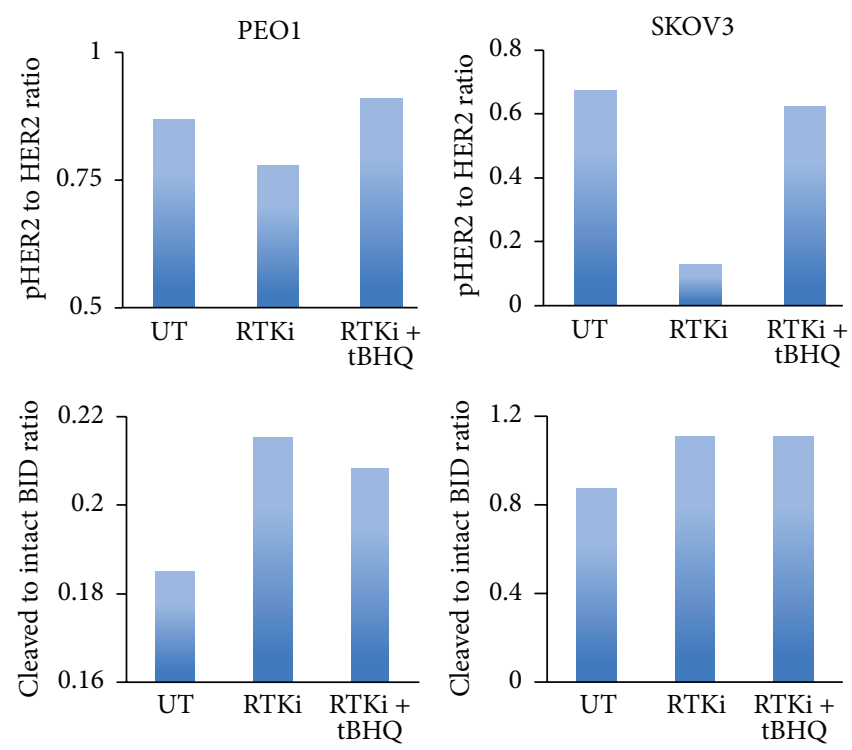

(b)

FIgURE 4: NRF2 activation desensitises RTK signaling pathway to HER2 inhibitors Pertuzumab and Trastuzumab. (a) Immunoblot analysis showing tBHQ dependent recovery of RTK signaling following its inhibition by HER2 inhibitors. Exponentially growing cells were either left untreated (UT) or treated with combination of HER2 inhibitors, Pertuzumab and Trastuzumab at concentration of $20 \mu \mathrm{g} / \mathrm{mL}$ (RTKi), or with cotreatment of $200 \mu \mathrm{M} \mathrm{tBHQ}(\mathrm{RTKi}+\mathrm{T})$ for $4 \mathrm{~h}$ before and processed for immunoblotting using relevant antibodies (Table 1). Ponceau stain of the same blot was used as loading control. (b) Bar chart showing total HER2, phospho-HER2, total HER3, phospho-AKT, phospho-ERK, phospho-NRF2, and BID levels in PEO1 and SKOV3 cell lines by quantifying immunoblot signal intensities obtained in (a) and normalised to the value of UT and expressed as fold change. Blue bars show ratio of phospho-HER2 to HER2 (upper panels) and cleaved BID to intact BID (lower panels).

protein levels of HER2 and HER3 (Figure 2) and we further confirmed that the protein upregulation was as a result of their transcriptional induction (Figure 3). These findings were important because such receptor induction attenuated the inhibitory responses of HER2-targeted drugs (Figure 4). As an alternative approach to study the regulation of HER receptors by NRF2, we knocked down NRF2 in our cell lines and firstly studied the protein levels of the receptors and their downstream substrate, pATK. We found significant protein repression of HER2 and HER3 as well as pAKT. Quantification of the resulting immunoblot signals revealed a greater repression with 75 pmol NRF2 SiRNA (Figure 7(a)). Interestingly, we could also detect and capture such repression at single cell level by performing immunostaining for HER2 and pAKT following either scrambled or NRF2 specific SiRNA (Figure 7(b)). Immunolabelling also revealed localisational features of total HER2 and pAKT. HER2 was mostly localised at the cell membrane as expected and apparently without any nuclear staining. The pAKT on the other hand was localised at the cell membrane, general cytosol and nucleus, as revealed by immunostaining and the merger with DAPI staining (Figure 7(b)). This is consistent with previous reports of nucleocytoplasmic shuttling of pATK that could have physiological consequences [63-65].

We next wanted to determine and confirm any transcriptional mechanism of NRF2 specific SiRNA dependent repression of HER2 and HER3. We thought this could be a likely explanation as we earlier showed tBHQ dependent transcriptional upregulation of HER receptors (Figure 3 ). To address this, we again utilised our transcriptional reporter assays for both HER2 and HER3 receptors that were established for this study. Using our ovarian cell line models, we individually transfected the reporter systems but, this time, cotransfecting with NRF2 specific SiRNA as well. Following this, cells were either left untreated or treated with $\mathrm{tBHQ}$. We found that following NRF2 knockdown, HER2 transcription was significantly repressed in both PEO1 (Figure $7(\mathrm{c})$ ) and SKOV3 (Figure 7(d)) cell lines. In terms of HER3, while NRF2 knockdown significantly repressed transcription in SKOV3, such repression was not seen in PEO1 (compare Figures $7(\mathrm{c})$ and 7 (d) for prHER3). Interestingly, the tBHQ dependent transcriptional induction of prHER 2 and prHER3 gene reporters as seen in Figure 4 was disrupted following depletion of NRF2 in both PEO1 and SKOV3 cell lines to significant levels (Figures $7(\mathrm{c})$ and $7(\mathrm{~d})$ ). These important findings confirmed that $\mathrm{tBHQ}$ mediated protein and transcriptional induction of HER receptors was dependent on NRF2 and not by any off NRF2 target effect of tBHQ treatment. Finally, we repeated knockdown of NRF2 either alone or with parallel knockdown of KEAP1 and exposed such cells to targeted immunotherapeutics for 24 and $48 \mathrm{~h}$ (Figure $7(\mathrm{e})$ ). We found significant increase in cell death in NRF2 knockdown cells upon exposure to the immunotherapeutics and significant reversal of this response with parallel KEAP1 knockdown.

These results confirmed the transcriptional regulatory role of NRF2 for HER2 and HER3 receptors and illustrated alteration of protein abundance as a result of such transcriptional regulation. These data also confirmed 


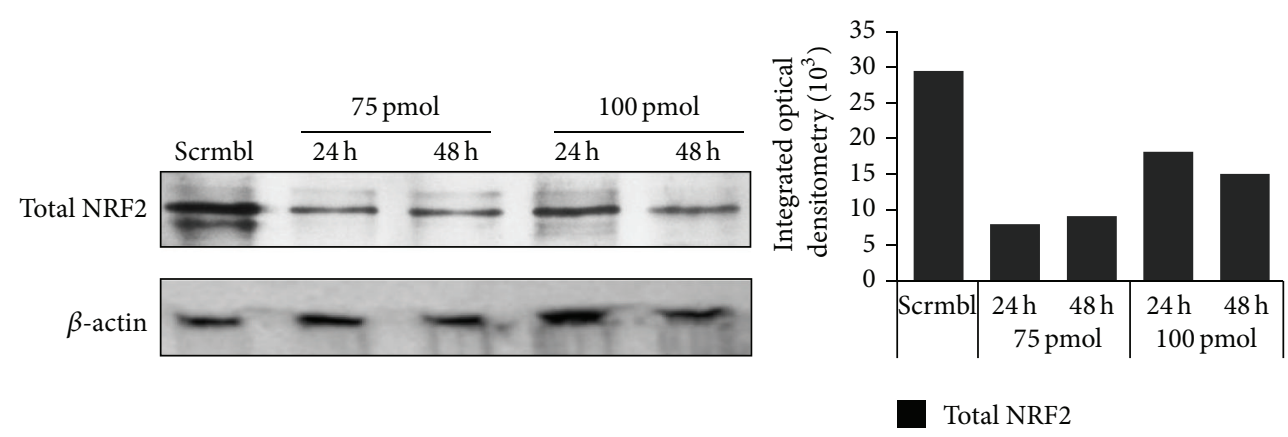

(a)
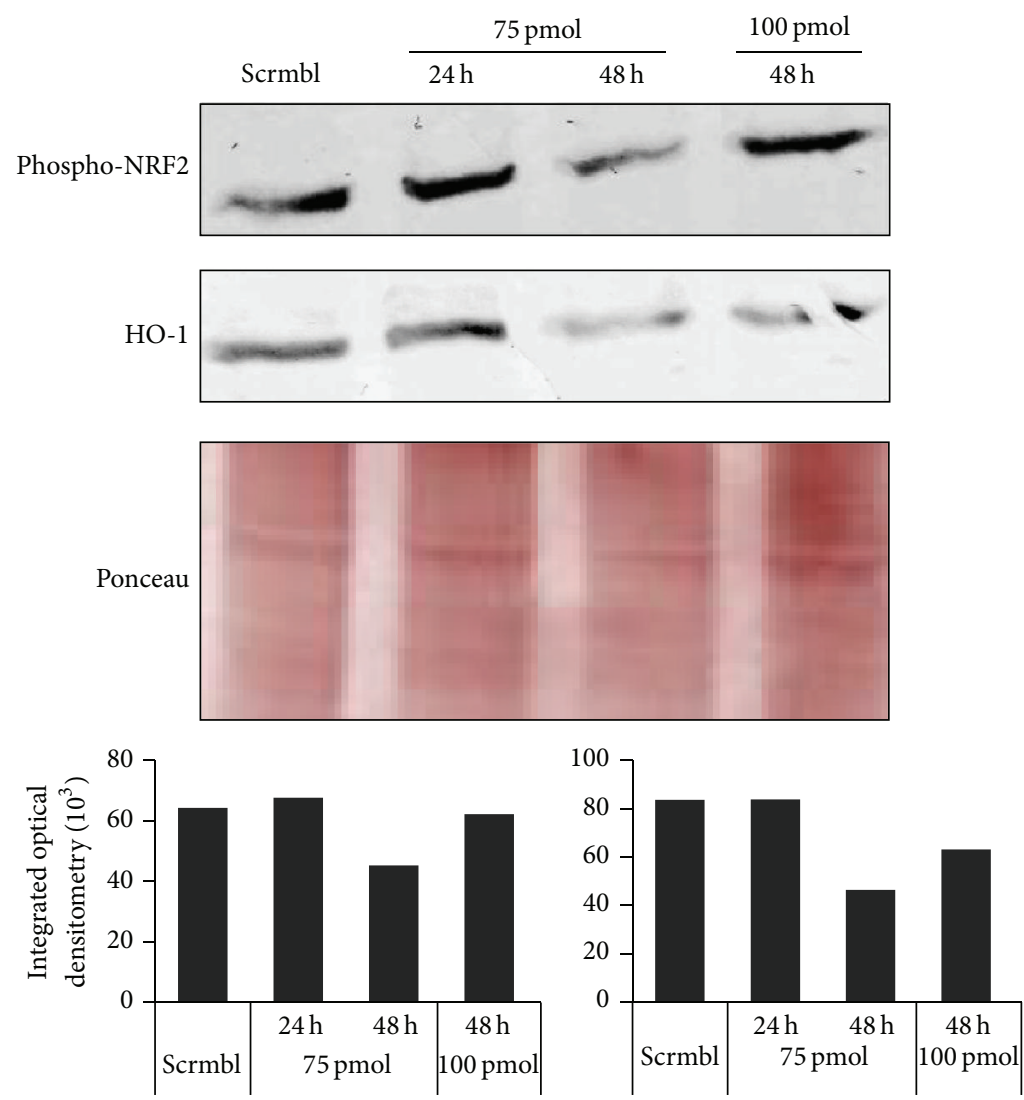

pNRF2

HO-1

(b)

ROS levels

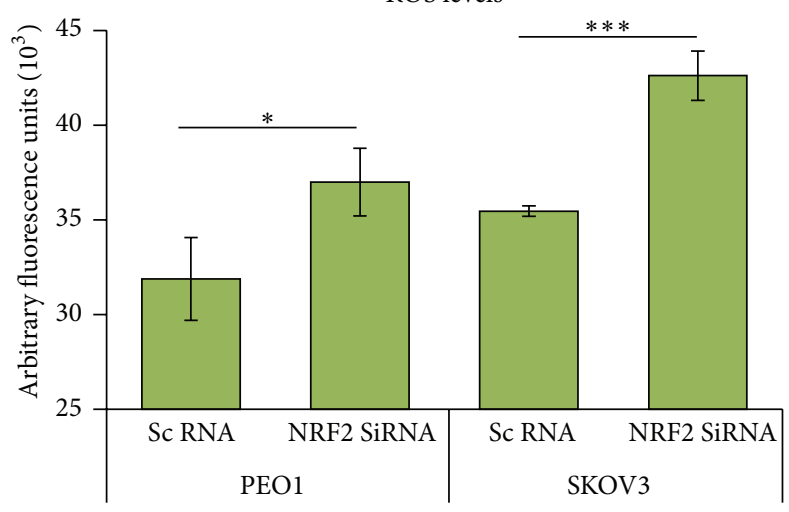

(c)

FIgure 5: Continued. 

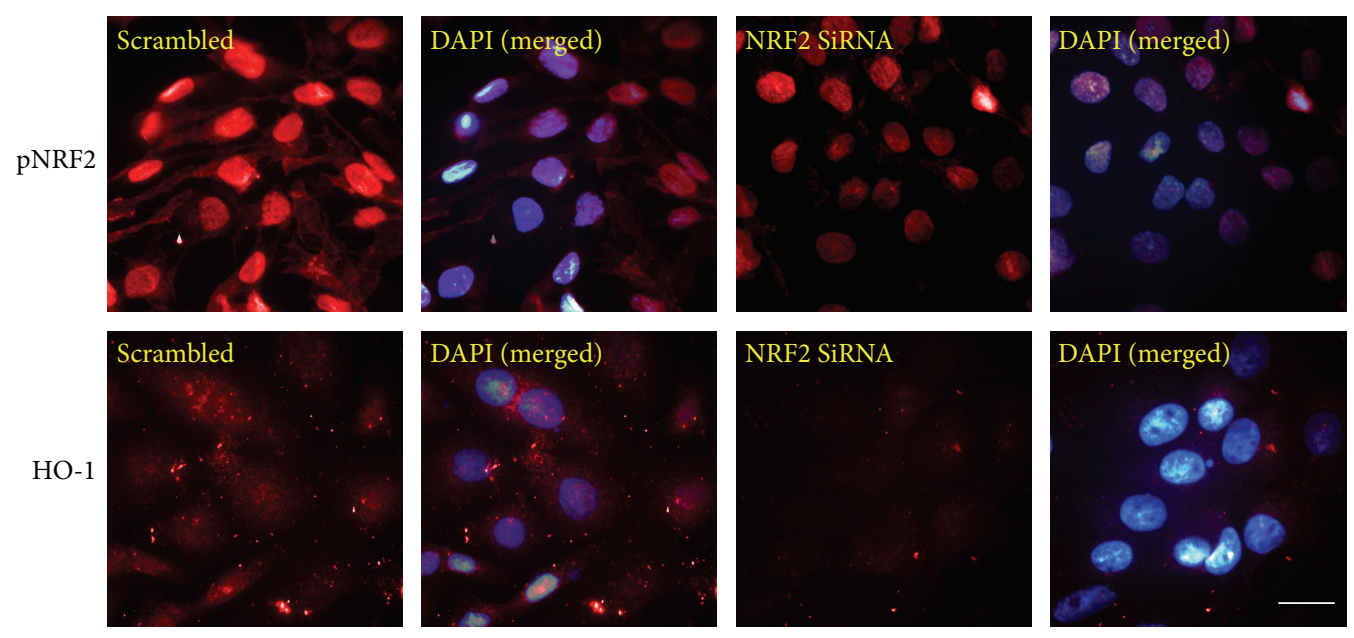

(d)

FIGURE 5: Knockdown of NRF2 by SiRNA causes repression of phospho-NRF2 and HO-1 levels and elevation of reactive oxygen species (ROS). (a) Optimization of SiRNA-mediated NRF2 knockdown. Exponentially growing cells were transfected either with scrambled RNA (scrmbl) or with different amounts of SiRNA for either $24 \mathrm{~h}$ or $48 \mathrm{~h}$ before being processed for immunoblotting. (b) NRF2 knockdown results in repression of its substrates. The same lysates as in (a) were blotted for phospho-NRF2 and HO-1 levels. Bar charts in (a) and (b) show total NRF2, phospho-NRF2, and HO-1 levels in SKOV3 cell lines by quantifying immunoblot signal intensities obtained in respective blots and normalised to the value of UT and expressed as fold change. (c) NRF2 knockdown leads to ROS accumulation. SKOV3 cells were seeded in triplicate for $18 \mathrm{~h}$ and transfected with NRF2 SiRNA. Following $48 \mathrm{~h}$ incubation, cells were assayed for total ROS by loading them with DCFDA for $45 \mathrm{~min}$ and measuring fluorescence using fluorescence multiplate reader (MODULUS, Promega) with excitation and emission spectra of $485 \mathrm{~nm} / 535 \mathrm{~nm}$. The fluorescence reading was normalised to total cell abundance within the same wells as described in Materials and Methods. Data are the means with \pm S.D. of triplicates, normalised to untreated (UT) control and expressed as fold change with statistical significance determined by Student's $t$-test according to the scale $*: p<0.05, * *: p<0.01$, and $* * *: p<0.001$. (d) Immunofluorescent labelling of endogenous phospho-NRF2 and HO-1 exhibits repression following NRF2 knockdown. Cells were transfected as in (a) and processed for immunocytochemistry. Relevant primary antibodies followed by Alexa Fluor conjugated secondary antibodies were used for immunolabelling for phospho-NRF2 and HO-1 (red fluorescence). Nuclear reference was provided by costaining with 4',6-Diamidino-2Phenylindole, Dihydrochloride (DAPI). Images were captured with Leica DMiRe2 electronic microscope with 100x objective while merging, colocalisation, and further analysis were performed by using integrated features of Andor iQ Core software (ANDOR Technologies Ltd.). Scale bar indicates $10 \mu \mathrm{m}$.

the role of NRF2 in determining overall treatment responses to HER2 targeting immunotherapeutics and hence defining the balance between resistance and sensitivity.

\section{Discussion}

The receptor tyrosine kinases (RTKs), exemplified by HER2/ HER3 family receptors, are key regulators of cellular proliferation, differentiation, and survival, as well as determinants of cancer initiation, maintenance, and progression [1-4]. Complexity in understanding the HER2/HER3 activation and signaling arises from the intricate and complex regulation of coexpression of HER2/HER3 receptors and their ligands and the broad spectrum of tumour biochemistry, heterogeneity, and range of sensitivities and resistance exhibited to drugs targeting the HER receptor system $[13,14,17$, 21]. Furthermore, clinical data on HER2/HER3 coexpression profile correlates to some degree with disease-free survival, not only regarding anti-RTK treatment outcome [66], but also by other therapeutic agents $[43,67,68]$. However, it has been suggested that sustained and complete inhibition of HER3 and its output to PI3K/Akt is required for the maximal antitumour effect of HER2 inhibitors $[13,16]$ and that inhibition of HER2 receptor alone might not generate sufficient anticancer response [16]. Lately, data have accrued to evidence and implicate NRF2 and ROS, in addition to HER2/HER3, in the promotion of cellular proliferation and therapeutic resistance in cancer cells $[31,69,70]$. It is also known that ROS can trigger both the AR and the HER family receptor pathways with concomitant transcriptional upregulation of HER2/HER3 and NRF2 and subsequent elevation and activation of their functions [31, 68-70]. Thus, the HER2/HER3 family receptor signaling pathway is upstream of PI3K/AKT/mTOR pathway [13-16] and has likewise been shown to be upstream of the NRF-AR pathway as well $[46,47$, $59,60,71,72]$. These highlight the possibility of a more direct, rather than indirect, contact and cross relationship between the HER2/HER3 and NRF2-AR pathways. This crosstalk could be likely and even necessary as RTK dependent growth and metabolism creates ROS, which would require parallel NRF2 dependent antioxidant pathway for its neutralisation. Likewise, the implication of NRF2 in proliferative and cytoprotective pathways may involve RTK dependent signaling [73]. RTK-targeted cancer therapies are compromised or limited when tumour cells circumvent the action of a single agent, and multiple agents due to the readjustments in 

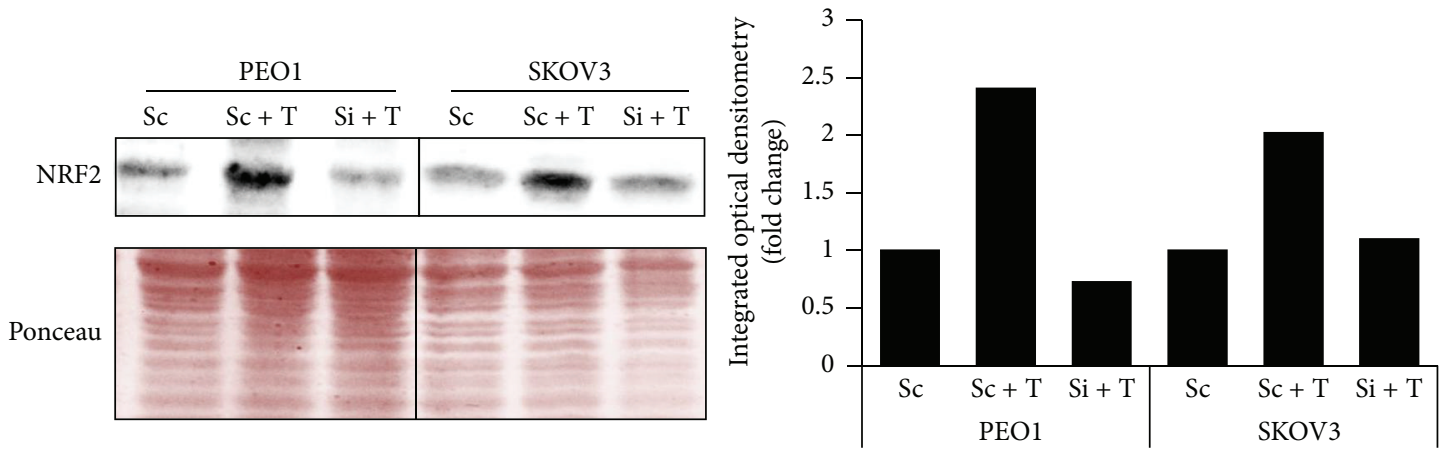

NRF2

(a)
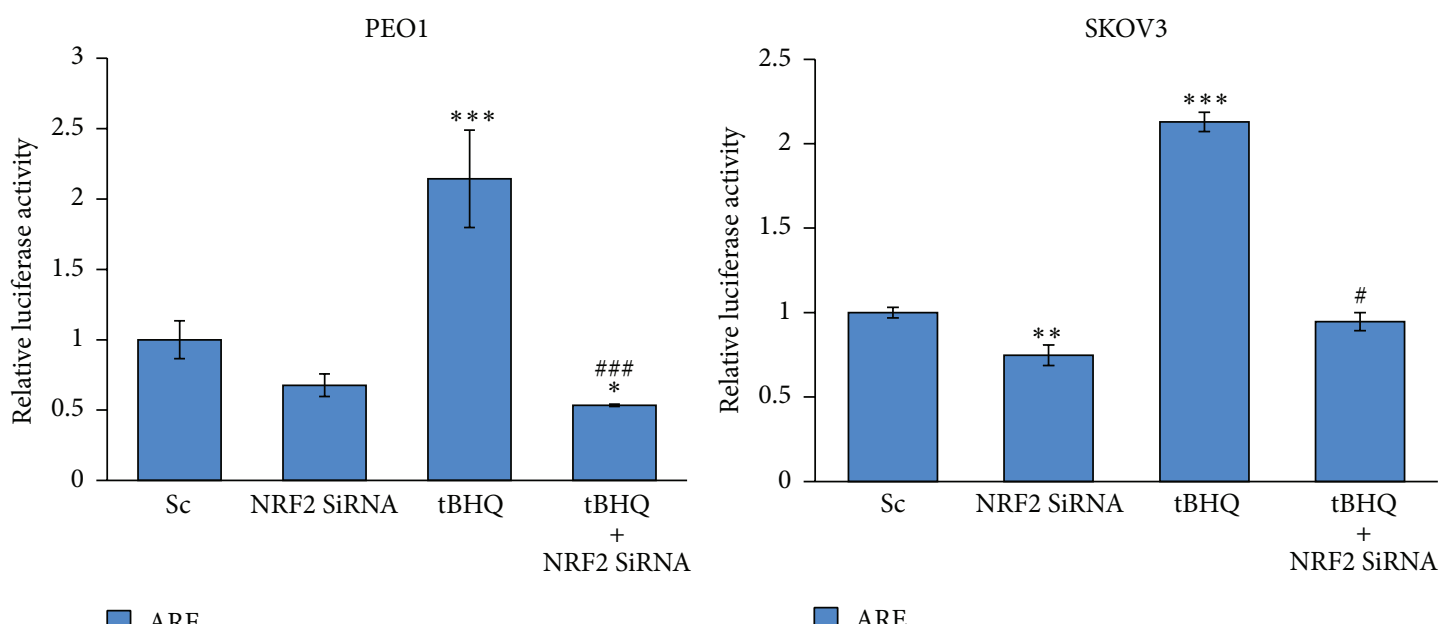

(b)

FIGURE 6: Knockdown of NRF2 by SiRNA represses both basal and induced antioxidant response pathway in PEO1 and SKOV3 cell lines. (a) Immunoblotting analysis showing repression of NRF2 following NRF2 knockdown by SiRNA in PEO1 and SKOV3 cell lines. Cells were either transfected with scrambled SiRNA (Sc) or transfected with 75 pmol of NRF2 SiRNA (Si). After 48 h, cells were either left untreated or treated with $200 \mu \mathrm{M}$ tBHQ (T) for $4 \mathrm{~h}$, before being processed for immunoblotting using relevant antibodies (Table 1). Ponceau stain of the same blot was used as loading control. Bar chart shows NRF2 levels by quantifying immunoblot signal intensities obtained in (a) and normalised to the value of untreated (UT) control and expressed as fold change. (b) Knockdown of NRF2 causes inhibition of its transcriptional antioxidant program in both constitutive and tBHQ induced states. PEO1 and SKOV3 cells were transfected with either empty PGL3 basic vector or $1 \mu \mathrm{g}$ PGL3 basic vector with a cloned $8 \mathrm{x}$ cis-antioxidant response elements (ARE) driving NRF2 dependent expression of luciferase gene. Cotransfection with $0.2 \mu \mathrm{g}$ pRL-CMV plasmid was performed as an internal transfection control. Where required, cotransfection with either scrambled RNA (Sc) or NRF2 SiRNA was performed using 20 pmol SiRNA. At $24 \mathrm{~h}$ after transfection, treatment with $200 \mu \mathrm{M}$ tBHQ was performed where indicated for $4 \mathrm{~h}$ following which, cells were processed for dual luciferase reporter assay (Promega) to record luciferase activity in multiplate reader (MODULUS, Promega). Data are the means with \pm S.D. of triplicates normalised to the value of scrambled SiRNA (Sc) and expressed as fold change with statistical significance determined by ONE WAY ANOVA followed by Tukey's post hoc test. * indicates significance of scramble versus treatment groups while \# indicates significance of tBHQ versus tBHQ + NRF2 SiRNA groups according to the scale symbolised by $*$ or $\#$ : $p<0.05, * *$ or \#\#: $p<0.01$, and $* * *$ or \#\#\#: $p<0.001$.

coexpression of HER2/HER3 receptors, their ligand binding dynamics, or changing preference for the dimerizing partner $[17,21,61,74,75]$ suggest that the anticancer effect of these agents might be further optimized or be better predicted by effectively limiting HER2/HER3 expression at the DNA level or at least identifying a common regulatory centre of HER2 and HER3 transcription. Thus the identification of factors that mediate or modulate the transcriptional expression of HER2/HER3 will be paramount.
NRF2 has already been implicated in numerous reports as a key contributor to resistance towards anticancer drugs. However, most of these past studies have explored the role of NRF2 in resistance against DNA damaging agents $[50,51,55]$. The present study demonstrates that NRF2 may regulate cancer cell proliferation, susceptibility, and resistance to targeted therapy via transcriptional regulation of HER2/HER3. To demonstrate the role of NRF2 in RTK signaling and thus in determining responses to targeted therapies, we used HER2 

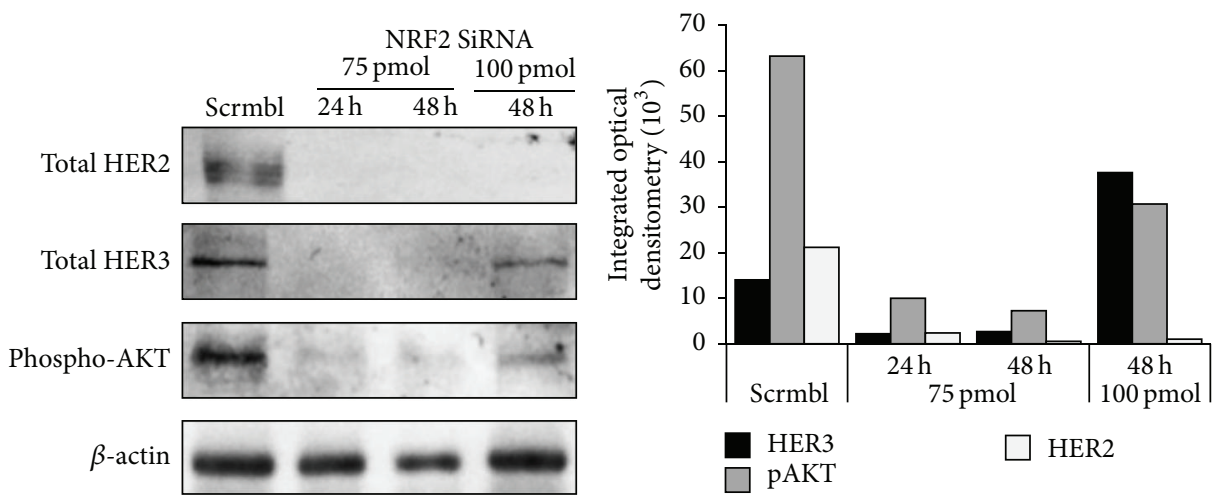

(a)
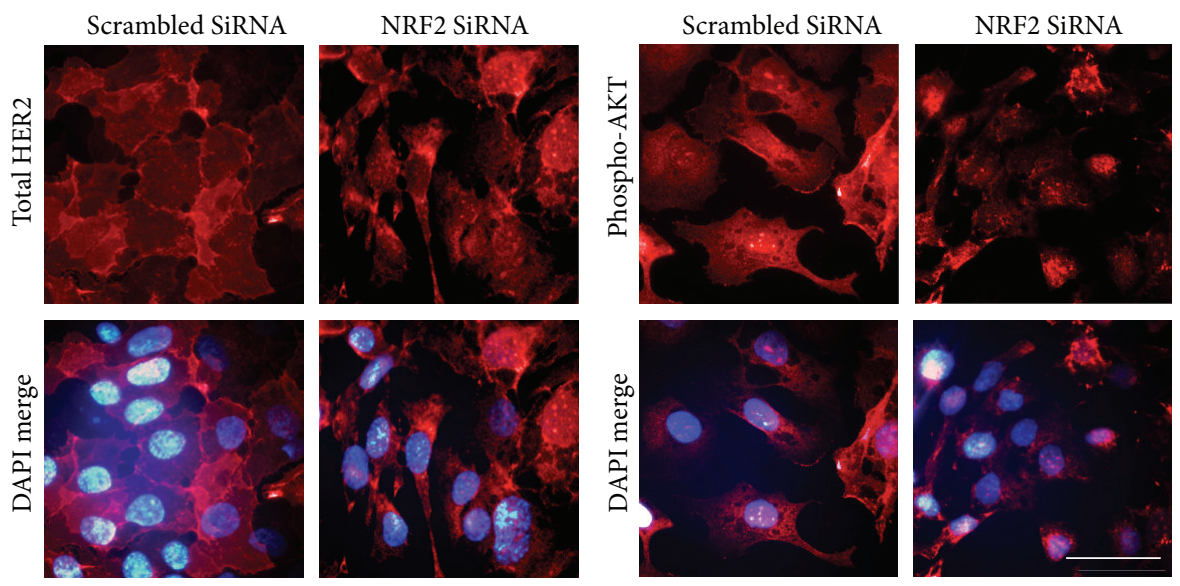

(b)

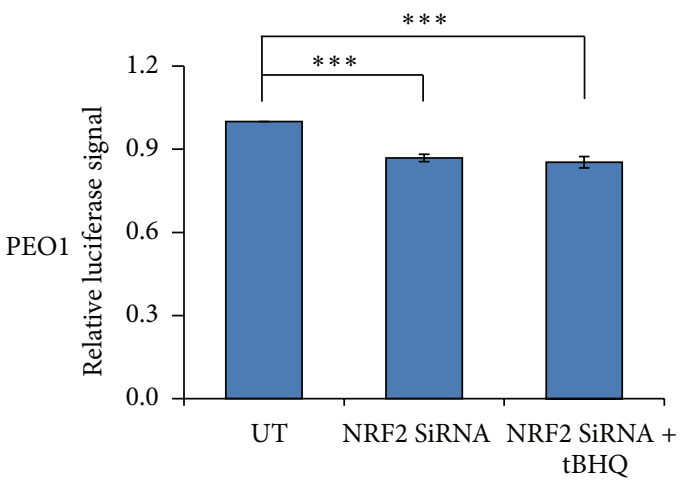

prHER2

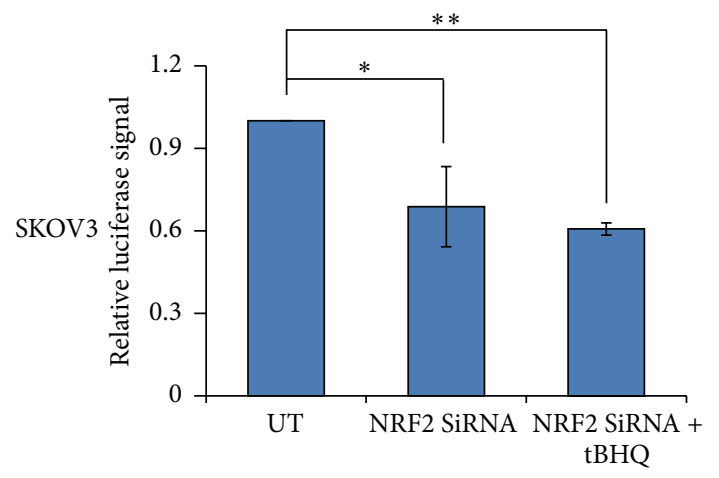

prHER2
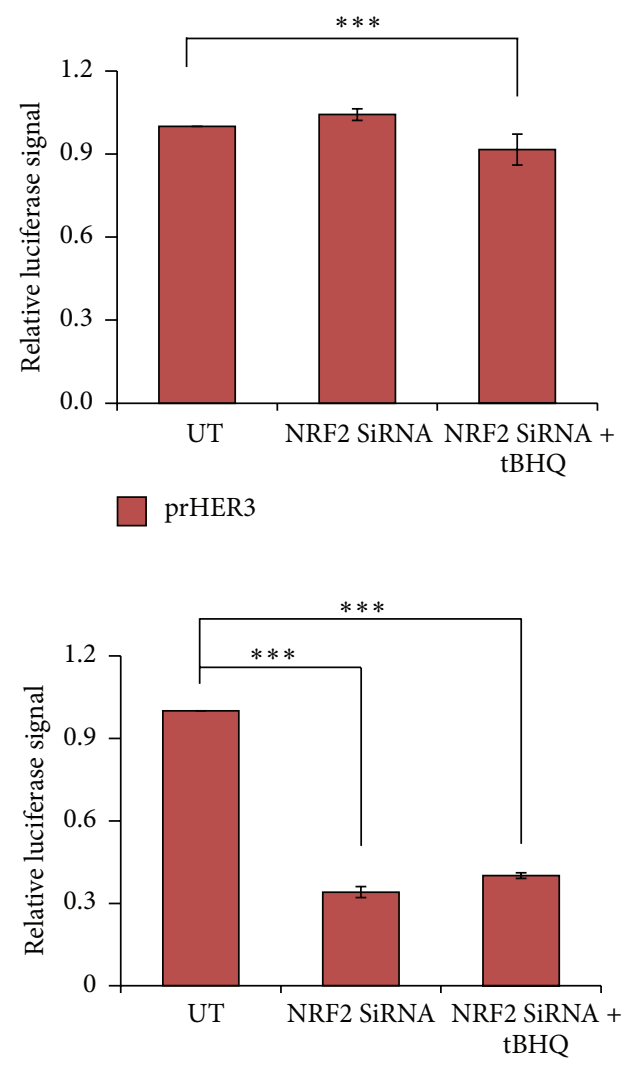

prHER3

(d) 


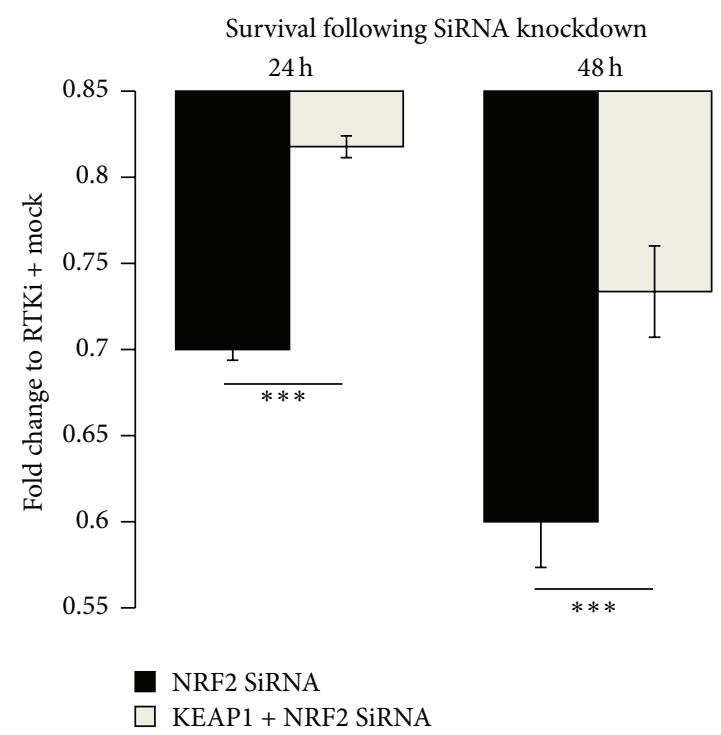

(e)

FIGURE 7: NRF2 knockdown causes downregulation of HER2 and HER3 levels, repression of pAKT, and sensitisation to targeted immunotherapeutics. (a) Immunoblotting analysis showing inhibition of RTK signaling following depletion of NRF2 mRNA by SiRNA in SKOV3 cell line. Exponentially growing cells were either transfected with scrambled SiRNA (Scrmbl) or transfected with 75 pmol of NRF2 SiRNA for either 24 or $48 \mathrm{~h}$ or $100 \mathrm{pmol}$ of NRF2 SiRNA for $48 \mathrm{~h}$ and processed for immunoblotting using relevant antibodies (Table 1). $\beta$ actin was used as a loading control. Bar chart shows protein levels by quantifying immunoblot signal intensities obtained and normalised to the value of untreated (UT) control and expressed as fold change. (b) Immunofluorescent labelling of endogenous total HER2 or phosphoAKT exhibits repression following NRF2 knockdown. Cells were transfected as in (a) and processed for immunocytochemistry. Relevant primary antibodies were used to stain HER2 or phospho-AKT followed by Alexa Fluor conjugated secondary antibody (red fluorescence). Nuclear reference was provided by costaining with $4^{\prime}, 6$-Diamidino-2-Phenylindole, Dihydrochloride (DAPI). Images were captured with Leica DMiRe2 electronic microscope at 100x objective while merging, colocalisation, and further analysis were performed by using integrated features of Andor iQ Core software (ANDOR Technologies Ltd.). Scale bar indicates $10 \mu \mathrm{m}$. (c and d) HER2 and HER3 downregulation following NRF2 knockdown is caused by their transcriptional repression. Exponentially growing PEO1 cells (c) or SKOV3 cells (d) were transfected with either empty PGL3 basic vector or $1 \mu \mathrm{g}$ PGL3 basic vector with cloned $1.5 \mathrm{~kb}$ fragments of either HER2 (prHER2) or HER3 (prHER3) upstream promoter regions driving the expression of luciferase gene. Cotransfection with $0.2 \mu \mathrm{g}$ pRL-CMV plasmid was performed as an internal transfection control. At $24 \mathrm{~h}$ after transfection, cells were either left untreated (UT) or treated with $200 \mu \mathrm{M}$ tBHQ as indicated for $4 \mathrm{~h}$ following which, cells were processed for dual luciferase reporter assay (Promega) to record luciferase activity in multiplate reader (MODULUS, Promega). (d) The same was done for SKOV3 cell lines. (e) Knockdown of NRF2 through SiRNA sensitises cancer cell to RTK inhibitors while parallel knockdown of KEAP1 partially relieves this sensitisation. Cells were transfected with scrambled SiRNA or SiRNA targeting NRF2 either alone or with the inclusion of KEAP1 SiRNA. Following further $24 \mathrm{~h}$ incubation, cells were either left untreated or treated with $25 \mu \mathrm{g} / \mathrm{mL}$ of HER2 inhibitors Pertuzumab and Trastuzumab. Cytotoxicity assay was performed as in (a). In (c-e), data are the means with \pm S.D. of triplicates and expressed as fold change with statistical significance determined by ONE WAY ANOVA followed by Tukey's post hoc test (for $\mathrm{c}$ and d), or Student's $t$-test (for e) according to the scale $*: p<0.05, * *: p<0.01$, and $* * *: p<0.001$.

overexpressing (SKOV3) and low expressing (PEO1) ovarian cancer cell lines [57] grown in HER receptor ligand Heregulin and employed pharmacological and genetic activation or inhibition of both NRF2-AR and HER2/HER3 signaling pathways.

Firstly, pharmacological activation of NRF2 with tBHQ enhanced ovarian cancer cell growth and protected cells from cytotoxicity caused by combined HER2-targeted immunotherapeutic agents, Pertuzumab and Trastuzumab. This was also concomitant with the induction of HER2, HER3, and pAKT proteins in oscillatory and dose-dependent fashions, which is consistent with current emerging concepts of transcriptional control and gene expression [70, 76-80]. Furthermore, NRF2 activation-dependent induction of the receptors and their signaling pathway was governed and executed by NRF2 at the transcriptional level of HER2 and
HER3 genes. Our results from both immunocytochemistry and gene reporter assays of HER2 and HER3 expressions were further supportive and reminiscent of recent reports that revealed that induction of HER2 might repress HER3 expression while its inhibition led to transcriptional induction of HER3 ([13, 14, 63], also see Figure 3(b)). It is clear that tBHQ treatment led to induction of NRF2, its associated antioxidant transcriptional program, and transcriptional and signaling activation of HER2 and HER3 and that this $\mathrm{tBHQ}$ response was evidently dependent on NRF2. Thus, NRF2 activation by tBHQ desensitised RTK signaling pathway to inhibitory action of the HER2 targeting immunotherapeutic agents Pertuzumab and Trastuzumab.

Next, to further investigate and confirm the involvement of NRF2 in the elevation of HER2 and HER3, we took a genetic approach to deplete NRF2 status and function using 


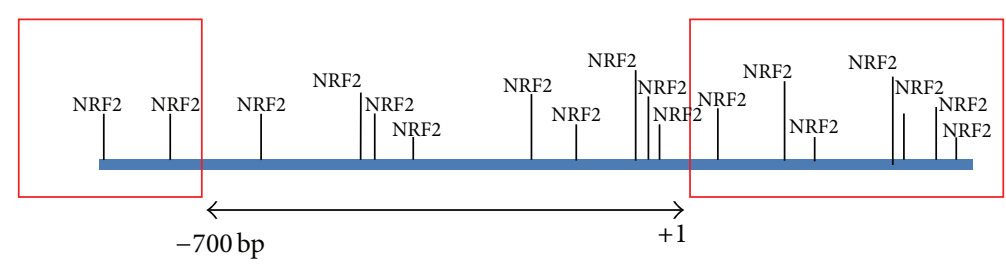

GCAAGAAGGGTGCATTTTGAAGAATTGAGATAGAAGTCTTTTTGGGCTGGGTGCAGTTGCTCGTGGTTG TAATTCCAGCACTTTGGGAGGCTGAGGCGGGAGGATCACCTGAGGTTGGGAGTTCAAGACCAGCCTCAC CAACGTGGAGAAACCCTGTCTTTACTAAAAATACAAAAAATTAGCTGGTCATGGTGGCACATGCCTGTA ATCCCAGCTGCTCGGGAGGCTGAGGCAGGAGAATCACTTGAACCAGGGAGGCAGAGGTTGTGGTGAGCA GAGATCGCGCCATTGCTCTCCAGCCTGGGCAACAAGAGCAAAAGTTCGTTTAAAAAAAAAAAAAAGTCC TTTCGATGTGACTGTCTCCTCCCAAATTTGTAGACCCTCTTAAGATCATGCTTTTCAGATACTTCAAAG ATTCCAGAAGATATGCCCCGGGGGTCCTGGAAGCCACAAGGTAAACACAACACATCCCCCTCCTTGACT ATCAATTTTACTAGAGGATGTGGTGGGAAAACCATTATTTGATATTAAAACAAATAGGCTTGGGATGGA GTAGGATGCAAGCTCCCCAGGAAAGTTTAAGATAAAACCTGAGACTTAAAAGGGTGTTAAGAGTGGCAG

$1.5 \mathrm{~kb}$ promoter CCTAGGGAATTTATCCCGGACTCCGGGGGAGGGGGCAGAGTCACCAGCCTCTGCATTTAGGGATTCTCC GAGGAAAAGTGTGAGAACGGCTGCAGGCAACCCAGGCGTCCCGGCGCTAGGAGGGACGCACCCAGGCCT GCGCGAAGAGAGGGAGAAAGTGAAGCTGGGAGTTGCCACTCCCAGACTTGTTGGAATGCAGTTGGAGGG GGCGAGCTGGGAGCGCGCTTGCTCCCAATCACAGGAGAAGGAGGAGGTGGAGGAGGAGGGCTGCTTGAG GAAGTATAAGAATGAAGTTGTGAAGCTGAGATTCCCCTCCATTGGGACCGGAGAAACCAGGGGAGCCCC CCGGGCAGCCGCGCGCCCCTTCCCACGGGGCCCTTTACTGCGCCGCGCGCCCGGCCCCCACCCCTCGCA GCACCCCGCGCCCCGCGCCCTCCCAGCCGGGTCCAGCCGGAGCCATGGGGCCGGAGCCGCAGTGAGCAC CATGGAGCTGGCGGCCTTGTGCCGCTGGGGGCTCCTCCTCGCCCTCTTGCCCCCCGGAGCCGCGAGCAC CCAAGGTGGGTCTGGTGTGGGGAGGGGACGGAGCAGCGGCGGGACCCTGCCCTGTGGATGCCCCGCCGA GGTCCCGCGGCCGGCGGGGCCAGAGGGGCCCGGACGAGCTCTCCTATCCCGAAGTTGTGGACAGTCGAG ACGCTCAGGGCAGCCGGGCCCTGGGGCCCTCGGGCGGGAGGGGGCAGTTACACGGCAGCGGCTCGAGAT GGCCCATCCAAGAGAC

(a) Putative transcription factor binding sites found along HER2 promoter

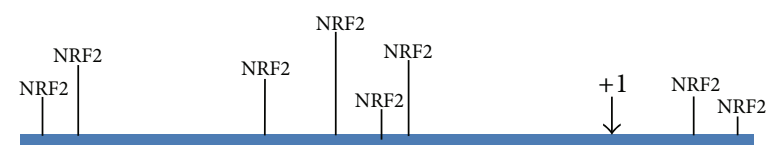

GCCCTCTAGGTTGCATATCAATAGGGAGCATGTTTAAGGAATGTTAGCCGGTAGTCTTTGCTAGGTGTG AGGGGTGAAATTTTTCTTTATCAAGGCTCAACTGTTTTCGAAGTCTTCAGGCTTGAAGTTCTGGAGAAA ACAACTAGGCTCTCCGGGCGAGATCCCGAATACCAGTTTAAGGGATTTGAAATGCAAGGCCGTCTGGGA CTCCACTGCCACGGATGGGCACCAGGCGGCGCCGGTCGGATCCGTCCCGGGACTAGCAGGGCTTTGGGC AGCAACCCGCAGGGAGCCCGACCGCCTCTGGCCAGGTCCGGGCAGCTGGTGGGGGAGGTTCCAGAGGTC CACGCCATTCGTGGACGCAGTCTCTAGTGTCCTCTCCGCGTCCCACTTCACTGCCCCATCCCCTTTCCT GCGAGAGCCTGGACTTGGAAGGCACCTGGGAGGGTGTAAGCGCCTTGGTGTGTGCCCATCTGGGTCCCC AGAAGAGCGGCGGGAACTGCGGCCGCCCGGACGGTGCGGCCAGACTCCAGTGTGGAAGGGGAGGCAGCT GTTCTCCCAGGCGGCCGTGGGGGGCAGCAGAGGGGACGGCGACAGGTGCGGGAGCCCCTCCCGGGGTAG AAGTGGAAAGGCGGGCTCCGGGGTCTGTTCCCAGGCTGGAAACCACCCCCGCCCCCCATCCAAATCCCC GGGAGAGGCCCGGCCGGCGCCGGGTCTGGAGGAGGAAGCGGCCAGAGACAGTGCAATTTCACGCGGTCT CTGTGGCTCGGGTTCCTGGGCTGGGTGGATGAATTATGGGGTTTCGAGTCTGGGAGAAACTGAGGTGGC CTGGACGTGAGGCAAAAAACACCCTCCCCCTCAAAAACACACAGAGAGAAATATTCACATTCTGAGAGA AAATCCACCAAGTGAACCAACCGGCTAGGGGAGTTGAGTGATTTGGTTAATGGGCGAGGCCAACTTTCA GGGGGCAGGGCTTTGGAGAGCTTTCCACTCCCTCATTCATTACCCTTCCCTGGATCTGGGGGCTTTCGG AATCTCGACCTCCCCTTGGCCTATCTCCTGCAGAAAAATTAGGGTGAGCCCCATCCTCGATCTGCTCCG CCAAGTTGCGGGACCGCGGGGCGTGGCACGCTCGGGGCAGGCGGTCCGAGGCTCCGCAATCCCTACTCC AGCCTCGCGCGGGAGGGGGCGCGGCCGTGACTCACCCCCTTCCCTCTGCGTTCCTCCCTCCCTCTCTCT CTCTCTCTCACACACACACACCCCTCCCCTGCCATCCCTCCCCGGACTCCGGCTCCGGCTCCGATTGCA ATTTGCAACCTCCGCTGCCGTCGCCGCAGCAGCCACCAATTCGCCAGCGGTTCAGGTGGCTCTTGCCTC GATGTCCTAGCCTAGGGGCCCCCGGGCCGGACTTGGCTGGGCTCCCTTCACCCTCTGCGGAGTCATGAG GGCGAACGACGCTCTGCAGGTGCTGGGCTTGCTTTTC

(b) Putative transcription factor binding sites found along HER3 promoter

FIGURE 8: In silico analysis of HER2 and HER3 promoter sequences. (a) $1.5 \mathrm{~kb}$ promoter region of HER2 gene was fetched from database (ensemble.org) and subjected to transcriptional factor binding prediction program (http://consite.genereg.net/) to predict for putative NRF2 binding sites as indicated. Line with arrowheads shows the $0.7 \mathrm{~kb}$ sequence of HER2 promoter [8], while regions enclosed in rectangles show additional sequences included and cloned in the PGL3 luciferase reporter vector (Promega) because of carrying additional NRF2 binding sites. (b) The same analyses were performed for HER3 promoter. In (a) and (b) +1 indicate the transcriptional start site, sequences highlighted in green show NRF2 binding sites as predicted by ConSite, and sequences in bold represent manual identification of putative NRF 2 binding sites based on ARE consensus sequence [9] while those highlighted in pink show overlapping NRF2 binding sites by the two methods mentioned above. 
SiRNA. This approach increased cellular ROS, repressed pNRF2 and HO-1 levels, and even disrupted the tBHQ dependent induction of our ARE reporter system (Figures 5 and 6). In addition, NRF2 depletion by SiRNA caused transcriptional repression of HER2 and HER3 leading to lowered expression of HER2, HER3, and pAKT proteins. As an alternative approach, we also cloned and overexpressed individually both NRF2 and KEAP1 genes in our cancer cell lines and found these in either cytoprotection or sensitisation to targeted therapies, respectively (data not shown). Moreover, we illustrated that while knockdown of NRF2 significantly sensitised ovarian cancer cells to targeted immunotherapy, parallel knockdown of KEAP1 reversed this sensitisation. These results support and confirmed our earlier inferred regulatory role of NRF2 in the transcription of HER 2 and HER3 receptors and its association with alteration of HER2 and HER3 proteins abundance. A recent study has suggested a similar role for NRF2 in regulating the expression of HER2 [73] but fell short of evidencing direct transcriptional regulation as shown in this study. To demonstrate transcriptional modulation of these receptors, we generated and utilised luciferase reporter assays of their proximal promoter sequencing spanning $1.5 \mathrm{~kb}$ regions. We performed in silico analysis of these upstream regulatory regions for the presence of NRF2 binding and ARE like consensus sequences and found a number of such binding sites (Figure 8). Moreover, a direct interaction of NRF2 and HER2 in regulating the expression of NRF2 target genes, including HO-1, via binding of the complex to the ARE of the target genes has been reported [45] which adds credence to our observed downregulation of HER2, HER3, and pAKT as well as HO-1 and pNRF2 levels following our SiRNAmediated depletion of NRF2. However, further experiments are necessary to confirm the role of NRF2 as a transcription factor for HER receptors.

Thus we have shown that NRF2 regulates HER2 and HER3 signaling pathway to modulate sensitivity to targeted therapies. This demonstrates that NRF2 activation is not only implicated in resistance to genotoxic agents as previously shown [55] but can also lead to resistance to immunotherapies involving Pertuzumab and Trastuzumab, whose actions are very specific to HER2 receptors and unrelated to antioxidant pathway until this study.

\section{Conclusion}

The effectiveness of current anticancer therapies that involve DNA damaging and ROS producing agents is limited, because of NRF2 dependent emergence of cellular resistance to genotoxic agents. On the other hand, targeted anticancer therapeutic agents, while being initially found to be promising, have their own limitations. These include predicting their action and outcome owing to their tight dependence on properties such as cell surface expression of receptors, their dimerizing preferences, presence of ligands, and dynamics of recycling/degradation. This study has found a novel node of regulation between the AR and RTK signaling pathway. As such, the central regulatory node that converges at transcription factor NRF2 presents itself as a very attractive drug target especially in both scenarios of resistance described above. We have presented evidence at the gene expression, protein induction, localisation, and cytotoxicity levels that the two pathways are coregulated and together predict and inform outcomes to targeted immunotherapies and that such responses could be controlled by modulating NRF2 function.

\section{Conflict of Interests}

The authors declare no conflict of interests.

\section{Acknowledgment}

This work was partly funded by The Northwood Trust.

\section{References}

[1] D. Yu, J. K. Wolf, M. Scanlon, J. E. Price, and M.-C. Hung, "Enhanced c-erbB-2/neu expression in human ovarian cancer cells correlates with more severe malignancy that can be suppressed by E1A," Cancer Research, vol. 53, no. 4, pp. 891-898, 1993.

[2] A. Lipton, L. Goodman, K. Leitzel et al., "HER3, p95HER2, and HER2 protein expression levels define multiple subtypes of HER2-positive metastatic breast cancer," Breast Cancer Research and Treatment, vol. 141, no. 1, pp. 43-53, 2013.

[3] K. Asrani, R. A. Keri, R. Galisteo et al., "The HER2- and heregulin b1 (HRG)-inducible TNFR superfamily member Fn14 promotes HRG-driven breast cancer cell migration, invasion, and MMP9 expression," Molecular Cancer Research, vol. 11, no. 4, pp. 393-404, 2013.

[4] L. M. Balz, K. Bartkowiak, A. Andreas et al., "The interplay of HER2/HER3/PI3K and EGFR/HER2/PLC- $\gamma 1$ signalling in breast cancer cell igration and dissemination," The Journal of Pathology, vol. 227, no. 2, pp. 234-244, 2012.

[5] I. Alroy and Y. Yarden, "The ErbB signaling network in embryogenesis and oncogenesis: signal diversification through combinatorial ligand-receptor interactions," FEBS Letters, vol. 410, no. 1, pp. 83-86, 1997.

[6] W. X. Schulze, L. Deng, and M. Mann, "Phosphotyrosine interactome of the ErbB-receptor kinase family," Molecular Systems Biology, vol. 1, article 0008, 2005.

[7] A. Citri and Y. Yarden, "EGF-ERBB signalling: towards the systems level," Nature Reviews Molecular Cell Biology, vol. 7, no. 7, pp. 505-516, 2006.

[8] H. C. Hurst, "Update on HER-2 as target for cancer therapy: the ERBB2 promoter and its exploitation for cancer treatment," Breast Cancer Research, vol. 3, no. 6, pp. 395-398, 2001.

[9] J.-M. Lee and J. A. Johnson, "An important role of Nrf2ARE pathway in the cellular defense mechanism," Journal of Biochemistry and Molecular Biology, vol. 37, no. 2, pp. 139-143, 2004.

[10] M.-C. Hung, X. Zhang, D.-H. Yan et al., "Aberrant expression of the c-erbB-2/neu protooncogene in ovarian cancer," Cancer Letters, vol. 61, no. 2, pp. 95-103, 1992.

[11] D. Graus-Porta, R. R. Beerli, J. M. Daly, and N. E. Hynes, "ErbB-2, the preferred heterodimerization partner of all ErbB receptors, is a mediator of lateral signaling," The EMBO Journal, vol. 16, no. 7, pp. 1647-1655, 1997. 
[12] S. Burden and Y. Yarden, "Neuregulins and their receptors: a versatile signaling module in organogenesis and oncogenesis," Neuron, vol. 18, no. 6, pp. 847-855, 1997.

[13] J. T. Garrett, M. G. Olivares, C. Rinehart et al., "Transcriptional and posttranslational up-regulation of HER3 (ErbB3) compensates for inhibition of the HER2 tyrosine kinase," Proceedings of the National Academy of Sciences of the United States of America, vol. 108, no. 12, pp. 5021-5026, 2011.

[14] A. Chakrabarty, V. Sánchez, M. G. Kuba, C. Rinehart, and C. L. Arteaga, "Feedback upregulation of HER3 (ErbB3) expression and activity attenuates antitumor effect of PI3K inhibitors," Proceedings of the National Academy of Sciences of the United States of America, vol. 109, no. 8, pp. 2718-2723, 2012.

[15] C. García-García, Y. H. Ibrahim, V. Serra et al., "Dual mTORC1/2 and HER2 blockade results in antitumor activity in preclinical models of breast cancer resistant to anti-HER2 therapy," Clinical Cancer Research, vol. 18, no. 9, pp. 2603-2612, 2012.

[16] J. T. Garrett, C. R. Sutton, M. G. Kuba, R. S. Cook, and C. L. Arteaga, "Dual blockade of HER2 in HER2-overexpressing tumor cells does not completely eliminate HER3 function," Clinical Cancer Research, vol. 19, no. 3, pp. 610-619, 2013.

[17] A. Goltsov, Y. Deeni, H. Khalil et al., "Systems analysis of druginduced receptor tyrosine kinase reprogramming following targeted mono- and combination anti-cancer therapy," Cells, vol. 3, no. 2, pp. 563-591, 2014.

[18] T. Holbro and N. E. Hynes, "ErbB receptors: directing key signaling networks throughout life," Annual Review of Pharmacology and Toxicology, vol. 44, pp. 195-217, 2004.

[19] N. Normanno, C. Bianco, L. Strizzi et al., "The ErbB receptors and their ligands in cancer: an overview," Current Drug Targets, vol. 6, no. 3, pp. 243-257, 2005.

[20] E. M. Bublil and Y. Yarden, "The EGF receptor family: spearheading a merger of signaling and therapeutics," Current Opinion in Cell Biology, vol. 19, no. 2, pp. 124-134, 2007.

[21] A. Goltsov, S. P. Langdon, G. Goltsov, D. J. Harrison, and J. Bown, "Customizing the therapeutic response of signaling networks to promote antitumor responses by drug combinations," Frontiers in Oncology, vol. 4, article 13, 2014.

[22] A. K. Macleod, M. Mcmahon, S. M. Plummer et al., "Characterization of the cancer chemopreventive NRF2-dependent gene battery in human keratinocytes: demonstration that the KEAP1NRF2 pathway, and not the BACH1-NRF2 pathway, controls cytoprotection against electrophiles as well as redox-cycling compounds," Carcinogenesis, vol. 30, no. 9, pp. 1571-1580, 2009.

[23] H. Chen, J. Li, H. Li et al., "Transcript profiling identifies dynamic gene expression patterns and an important role for Nrf2/Keapl pathway in the developing mouse esophagus," PLoS ONE, vol. 7, no. 5, Article ID e36504, 2012.

[24] J. D. Hayes and A. T. Dinkova-Kostova, "The Nrf2 regulatory network provides an interface between redox and intermediary metabolism," Trends in Biochemical Sciences, vol. 39, no. 4, pp. 199-218, 2014.

[25] P. J. Meakin, S. Chowdhry, R. S. Sharma et al., "Susceptibility of Nrf2-null mice to steatohepatitis and cirrhosis upon consumption of a high-fat diet is associated with oxidative stress, perturbation of the unfolded protein response, and disturbance in the expression of metabolic enzymes but not with insulin resistance," Molecular and Cellular Biology, vol. 34, no. 17, pp. 3305-3320, 2014.

[26] M. Kimura, T. Yamamoto, J. Zhang et al., "Molecular basis distinguishing the DNA binding profile of Nrf2-Maf heterodimer from that of Maf homodimer," The Journal of Biological Chemistry, vol. 282, no. 46, pp. 33681-33690, 2007.

[27] W. Li, S. Yu, T. Liu et al., "Heterodimerization with small Maf proteins enhances nuclear retention of Nrf2 via masking the NESzip motif," Biochimica et Biophysica Acta-Molecular Cell Research, vol. 1783, no. 10, pp. 1847-1856, 2008.

[28] J. W. Kaspar, S. K. Niture, and A. K. Jaiswal, "Nrf2:INrf2 (Keap1) signaling in oxidative stress," Free Radical Biology and Medicine, vol. 47, no. 9, pp. 1304-1309, 2009.

[29] M. Kobayashi and M. Yamamoto, "Molecular mechanisms activating the Nrf2-Keapl pathway of antioxidant gene regulation," Antioxidants and Redox Signaling, vol. 7, no. 3-4, pp. 385-394, 2005.

[30] L. I. Gordon, M. A. Burke, A. T. K. Singh et al., "Blockade of the erbB2 receptor induces cardiomyocyte death through mitochondrial and reactive oxygen species-dependent pathways," The Journal of Biological Chemistry, vol. 284, no. 4, pp. 20802087, 2009.

[31] J. He, Q. Xu, Y. Jing et al., "Reactive oxygen species regulate ERBB2 and ERBB3 expression via miR-199a/125b and DNA methylation," EMBO Reports, vol. 13, no. 12, pp. 1116-1122, 2012.

[32] N. M. Reddy, S. R. Kleeberger, M. Yamamoto et al., "Genetic dissection of the Nrf2-dependent redox signaling-regulated transcriptional programs of cell proliferation and cytoprotection," Physiological Genomics, vol. 32, no. 1, pp. 74-81, 2007.

[33] Y. Deeni, H. Khalil, A. Goltsov, S. Langdon, D. Harrison, and J. Bown, "Quantitative analysis of proliferation behaviour of ovarian cancer cells with the dynamics of reactive oxygen species production and sequestration," Journal of Biotechnology, vol. 185, p. S12, 2014.

[34] H. S. Khalil, A. Goltsov, S. P. Langdon, D. J. Harrison, J. Bown, and Y. Deeni, "Quantitative analysis of NRF2 pathway reveals key elements of the regulatory circuits underlying antioxidant response and proliferation of ovarian cancer cells," Journal of Biotechnology, vol. 202, pp. 12-30, 2015.

[35] N. Wakabayashi, K. Itoh, J. Wakabayashi et al., "Keapl-null mutation leads to postnatal lethality due to constitutive Nrf2 activation," Nature Genetics, vol. 35, no. 3, pp. 238-245, 2003.

[36] T. Shibata, T. Ohta, K. I. Tong et al., "Cancer related mutations in NRF2 impair its recognition by Keap1-Cul3 E3 ligase and promote malignancy," Proceedings of the National Academy of Sciences of the United States of America, vol. 105, no. 36, pp. 13568-13573, 2008.

[37] T. Ohta, K. Iijima, M. Miyamoto et al., "Loss of Keap1 function activates Nrf2 and provides advantages for lung cancer cell growth," Cancer Research, vol. 68, no. 5, pp. 1303-1309, 2008.

[38] S. Homma, Y. Ishii, Y. Morishima et al., "Nrf2 enhances cell proliferation and resistance to anticancer drugs in human lung cancer," Clinical Cancer Research, vol. 15, no. 10, pp. 3423-3432, 2009.

[39] A. Lister, T. Nedjadi, N. R. Kitteringham et al., "Nrf2 is overexpressed in pancreatic cancer: implications for cell proliferation and therapy," Molecular Cancer, vol. 13, no. 10, article 37, 2011.

[40] M. Selvakumaran, D. A. Pisarcik, R. Bao, A. T. Yeung, and T. C. Hamilton, "Enhanced cisplatin cytotoxicity by disturbing the nucleotide excision repair pathway in ovarian cancer cell lines," Cancer Research, vol. 63, no. 6, pp. 1311-1316, 2003.

[41] T. Jiang, N. Chen, F. Zhao et al., "High levels of Nrf2 determine chemoresistance in type II endometrial cancer," Cancer Research, vol. 70, no. 13, pp. 5486-5496, 2010. 
[42] P. A. Konstantinopoulos, D. Spentzos, E. Fountzilas et al., "Keapl mutations and Nrf2 pathway activation in epithelial ovarian cancer," Cancer Research, vol. 71, no. 15, pp. 5081-5089, 2011.

[43] S. Wang, X. Huang, C.-K. Lee, and B. Liu, "Elevated expression of erbB3 confers paclitaxel resistance in erbB2-overexpressing breast cancer cells via upregulation of Survivin," Oncogene, vol. 29, no. 29, pp. 4225-4236, 2010.

[44] M.-I. Kang, A. Kobayashi, N. Wakabayashi, S.-G. Kim, and M. Yamamoto, "Scaffolding of Keap1 to the actin cytoskeleton controls the function of Nrf2 as key regulator of cytoprotective phase 2 genes," Proceedings of the National Academy of Sciences of the United States of America, vol. 101, no. 7, pp. 2046-2051, 2004.

[45] H. J. Kang, Y. W. Yi, Y. B. Hong et al., "HER2 confers drug resistance of human breast cancer cells through activation of NRF2 by direct interaction," Scientific Reports, vol. 4, p. 7201, 2014.

[46] L. M. Zipper and R. T. Mulcahy, "Inhibition of ERK and p38 MAP Kinases inhibits binding of Nrf2 and induction of GCS genes," Biochemical and Biophysical Research Communications, vol. 278, no. 2, pp. 484-492, 2000.

[47] K. W. Kang, J. I. H. Ryu, and S. G. Kim, “The essential role of phosphatidylinositol 3-kinase and of p38 mitogen-activated protein kinase activation in the antioxidant response elementmediated rGSTA2 induction by decreased glutathione in H4IIE hepatoma cells," Molecular Pharmacology, vol. 58, no. 5, pp. 1017-1025, 2000.

[48] M. Aslan and T. Özben, "Oxidants in receptor tyrosine kinase signal transduction pathways," Antioxidants \& Redox Signaling, vol. 5, no. 6, pp. 781-788, 2003.

[49] T. Nguyen, P. Nioi, and C. B. Pickett, "The Nrf2-antioxidant response element signaling pathway and its activation by oxidative stress," Journal of Biological Chemistry, vol. 284, no. 20, pp. 13291-13295, 2009.

[50] S. A. Rushworth, K. M. Bowles, and D. J. MacEwan, "High basal nuclear levels of Nrf2 in acute myeloid leukemia reduces sensitivity to proteasome inhibitors," Cancer Research, vol. 71, no. 5, pp. 1999-2009, 2011.

[51] G.-S. Shim, S. Manandhar, D.-H. Shin, T.-H. Kim, and M.K. Kwak, "Acquisition of doxorubicin resistance in ovarian carcinoma cells accompanies activation of the NRF2 pathway," Free Radical Biology and Medicine, vol. 47, no. 11, pp. 1619-1631, 2009.

[52] S. K. Niture and A. K. Jaiswal, "Nrf2-induced antiapoptotic Bcl-xL protein enhances cell survival and drug resistance," Free Radical Biology and Medicine, vol. 57, pp. 119-131, 2013.

[53] Y. Mitsuishi, K. Taguchi, Y. Kawatani et al., "Nrf2 redirects glucose and glutamine into anabolic pathways in metabolic reprogramming," Cancer Cell, vol. 22, no. 1, pp. 66-79, 2012.

[54] N. Morito, K. Yoh, K. Itoh et al., "Nrf2 regulates the sensitivity of death receptor signals by affecting intracellular glutathione levels," Oncogene, vol. 22, no. 58, pp. 9275-9281, 2003.

[55] X.-J. Wang, Z. Sun, N. F. Villeneuve et al., "Nrf2 enhances resistance of cancer cells to chemotherapeutic drugs, the dark side of Nrf2," Carcinogenesis, vol. 29, no. 6, pp. 1235-1243, 2008.

[56] A. H. Sims, A. J. M. Zweemer, Y. Nagumo et al., "Defining the molecular response to trastuzumab, pertuzumab and combination therapy in ovarian cancer," British Journal of Cancer, vol. 106, no. 11, pp. 1779-1789, 2012.
[57] S. P. Langdon, S. S. Lawrie, F. G. Hay et al., "Characterization and properties of nine human ovarian adenocarcinoma cell lines," Cancer Research, vol. 48, no. 21, pp. 6166-6172, 1988.

[58] C. Wallasch, F. U. Weiss, G. Niederfellner, B. Jallal, W. Issing, and A. Ullrich, "Heregulin-dependent regulation of HER2/neu oncogenic signaling by heterodimerization with HER3," The EMBO Journal, vol. 14, no. 17, pp. 4267-4275, 1995.

[59] R. Yu, C. Chen, Y.-Y. Mo et al., "Activation of mitogen-activated protein kinase pathways induces antioxidant response elementmediated gene expression via a Nrf2-dependent mechanism," The Journal of Biological Chemistry, vol. 275, no. 51, pp. 3990739913, 2000.

[60] J.-M. Lee, J. M. Hanson, W. A. Chu, and J. A. Johnson, "Phosphatidylinositol 3-kinase, not extracellular signal-regulated kinase, regulates activation of the antioxidant-responsive element in IMR-32 human neuroblastoma cells," The Journal of Biological Chemistry, vol. 276, no. 23, pp. 20011-20016, 2001.

[61] Y. Nagumo, D. Faratian, P. Mullen, D. J. Harrison, M. Hasmann, and S. P. Langdon, "Modulation of HER3 is a marker of dynamic cell signaling in ovarian cancer: implications for pertuzumab sensitivity," Molecular Cancer Research, vol. 7, no. 9, pp. 15631571, 2009.

[62] D. J. Slamon, W. Godolphin, L. A. Jones et al., "Studies of the HER-2/neu proto-oncogene in human breast and ovarian cancer," Science, vol. 244, no. 4905, pp. 707-712, 1989.

[63] R. Torka, K. Pénzes, S. Gusenbauer et al., "Activation of HER3 interferes with antitumor effects of Axl receptor tyrosine kinase inhibitors: suggestion of combination therapy," Neoplasia, vol. 16, no. 4, pp. 301-318, 2014.

[64] A. M. Martelli, G. Tabellini, D. Bressanin et al., "The emerging multiple roles of nuclear Akt," Biochimica et Biophysica ActaMolecular Cell Research, vol. 1823, no. 12, pp. 2168-2178, 2012.

[65] M. Rosner, M. Hanneder, A. Freilinger, and M. Hengstschläger, "Nuclear/cytoplasmic localization of Akt activity in the cell cycle," Amino Acids, vol. 32, no. 3, pp. 341-345, 2007.

[66] W. Xia, E. F. Petricoin III, S. Zhao et al., "An heregulin-EGFRHER3 autocrine signaling axis can mediate acquired lapatinib resistance in HER2+ breast cancer models," Breast Cancer Research, vol. 15, no. 5, article R85, 2013.

[67] B. Liu, D. Ordonez-Ercan, Z. Fan, S. M. Edgerton, X. Yang, and A. D. Thor, "Downregulation of erbB3 abrogates erbB2mediated tamoxifen resistance in breast cancer cells," International Journal of Cancer, vol. 120, no. 9, pp. 1874-1882, 2007.

[68] T. Shibata, S. Saito, A. Kokubu, T. Suzuki, M. Yamamoto, and S. Hirohashi, "Global downstream pathway analysis reveals a dependence of oncogenic NF-E2-Related factor 2 mutation on the mTOR growth signaling pathway," Cancer Research, vol. 70, no. 22, pp. 9095-9105, 2010.

[69] H. Sasaki, A. Suzuki, M. Shitara et al., "Keap1 mutations in lung cancer patients," Oncology Letters, vol. 6, no. 3, pp. 719-721, 2013.

[70] M. Lauriola, Y. Enuka, A. Zeisel et al., "Diurnal suppression of EGFR signalling by glucocorticoids and implications for tumour progression and treatment," Nature Communications, vol. 5, article 5073, 2014.

[71] C. Gorrini, B. P. Gang, C. Bassi et al., "Estrogen controls the survival of BRCA1-deficient cells via a PI3K-NRF2-regulated pathway," Proceedings of the National Academy of Sciences of the United States of America, vol. 111, no. 12, pp. 4472-4477, 2014.

[72] F. Rizvi, S. Shukla, and P. Kakkar, "Essential role of $\mathrm{PH}$ domain and leucine-rich repeat protein phosphatase 2 in Nrf2 suppression via modulation of Akt/GSK3b/Fyn kinase axis 
during oxidative hepatocellular toxicity," Cell Death \& Disease, vol. 5, no. 3, Article ID el153, 2014.

[73] S. Manandhar, B.-H. Choi, K.-A. Jung et al., "NRF2 inhibition represses ErbB2 signaling in ovarian carcinoma cells: implications for tumor growth retardation and docetaxel sensitivity," Free Radical Biology and Medicine, vol. 52, no. 9, pp. 1773-1785, 2012.

[74] B. K. Choi, X. Fan, H. Deng, N. Zhang, and Z. An, "ERBB3 (HER3) is a key sensor in the regulation of ERBB-mediated signaling in both low and high ERBB2 (HER2) expressing cancer cells," Cancer Medicine, vol. 1, no. 1, pp. 28-38, 2012.

[75] N. Gaborit, A. Abdul-Haia, M. Mancini et al., "Examination of HER3 targeting in cancer using monoclonal antibodies," Proceedings of the National Academy of Sciences of the United States of America, vol. 112, no. 3, pp. 839-844, 2015.

[76] Y.-Q. Xu, D. Zhang, T. Jin et al., "Diurnal variation of hepatic antioxidant gene expression in mice," PLoS ONE, vol. 7, no. 8, Article ID e44237, 2012.

[77] V. Pekovic-Vaughan, J. Gibbs, H. Yoshitane et al., "The circadian clock regulates rhythmic activation of the NRF2/glutathionemediated antioxidant defense pathway to modulate pulmonary fibrosis," Genes and Development, vol. 28, no. 6, pp. 548-560, 2014.

[78] H. Okazaki, N. Matsunaga, T. Fujioka et al., "Circadian regulation of mTOR by the ubiquitin pathway in renal cell carcinoma," Cancer Research, vol. 74, no. 2, pp. 543-551, 2014.

[79] D. Ohshima, K. Ichikawa, and C. Shih, "Regulation of nuclear NF- $\kappa$ B oscillation by a diffusion coefficient and its biological implications," PLoS ONE, vol. 9, no. 10, Article ID e109895, 2014.

[80] H. Shimojo, Y. Harima, and R. Kageyama, "Visualization of notch signaling oscillation in cells and tissues," Methods in Molecular Biology, vol. 1187, pp. 169-179, 2014. 


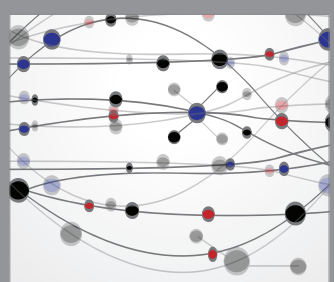

The Scientific World Journal
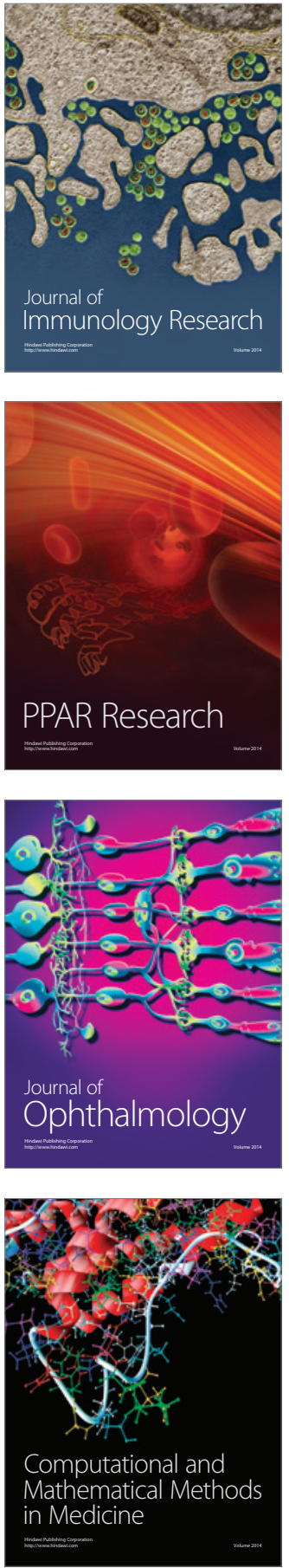

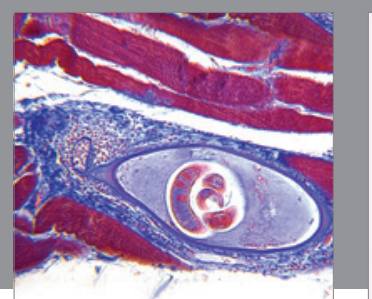

Gastroenterology Research and Practice

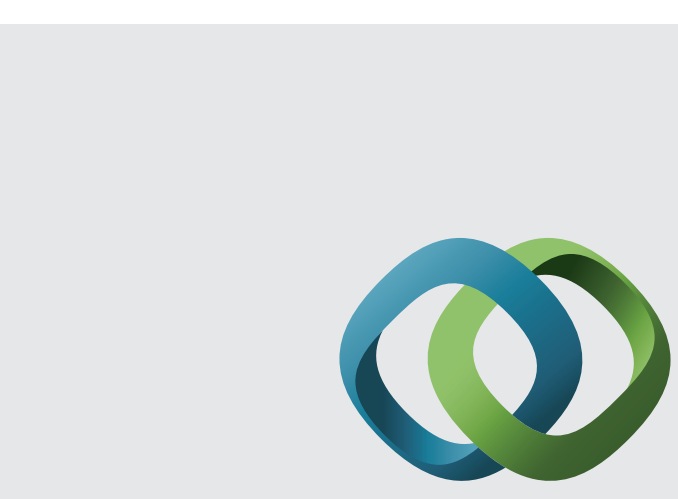

\section{Hindawi}

Submit your manuscripts at

http://www.hindawi.com
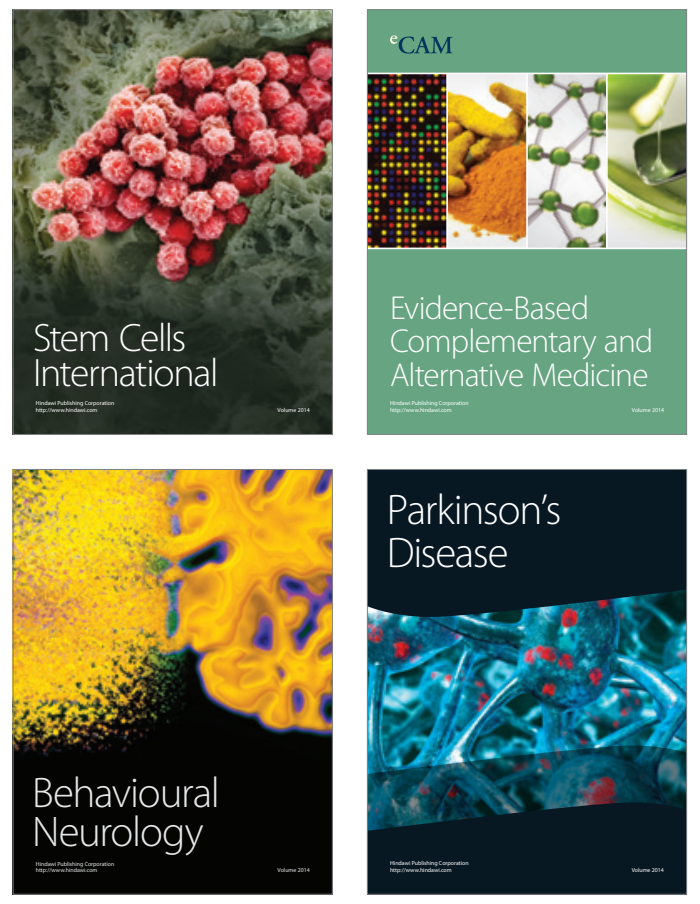
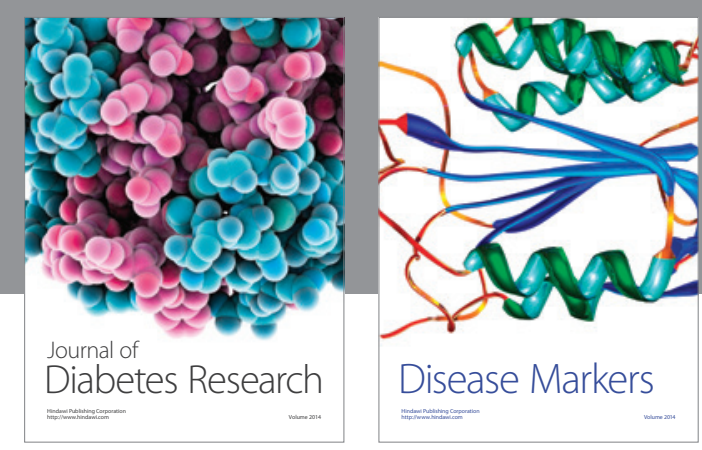

Disease Markers
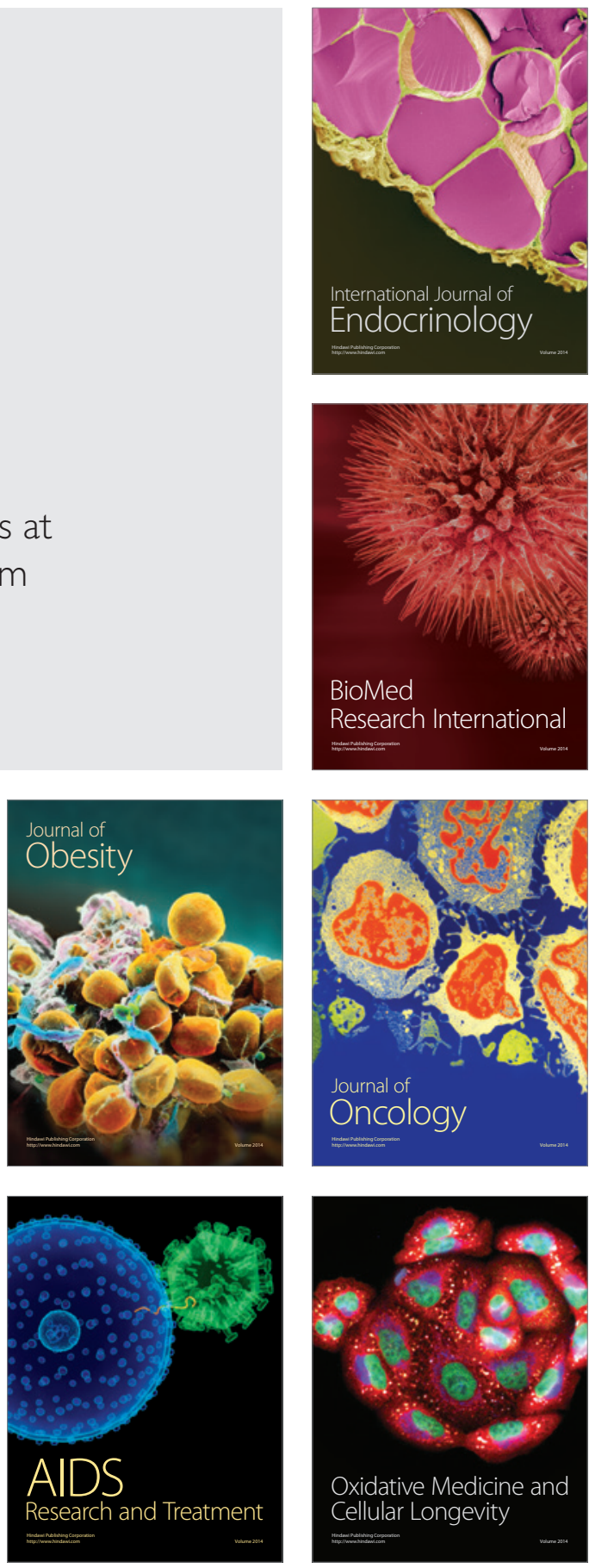